\title{
Structure of the magnetoionic medium around the Fanaroff-Riley Class I radio galaxy $3 \mathrm{C} 449$
}

\author{
D. Guidetti ${ }^{1,2,3}$, R. A. Laing ${ }^{1}$, M. Murgia ${ }^{4}$, F. Govoni ${ }^{4}$, L. Gregorini ${ }^{3}$, and P. Parma ${ }^{2}$ \\ ${ }^{1}$ European Southern Observatory, Karl-Schwarzschild-Straße 2, 85748 Garching, Germany \\ e-mail: dguidett@eso.org \\ 2 INAF - Istituto di Radioastronomia, bia Gobetti 101, 40129 Bologna, Italy \\ 3 Dipartimento di Astronomia, Univ. Bologna, bia Ranzani 1, 40127 Bologna, Italy \\ 4 INAF - Osservatorio Astronomico di Cagliari, Loc. Poggio dei Pini, Strada 54, 09012 Capoterra (CA), Italy \\ Received 14 December 2009 / Accepted 3 February 2010
}

\section{ABSTRACT}

\begin{abstract}
Aims. The goal of this work is to constrain the strength and structure of the magnetic field associated with the environment of the radio source 3C 449 by using observations of Faraday rotation, which we model with a structure function technique, and by comparison with numerical simulations. We assume that the magnetic field is a Gaussian isotropic random variable and that it is embedded in the hot intra-group plasma surrounding the radio source.

Methods. For this purpose, we present detailed rotation measure images for the polarized radio source 3C 449, previously observed with the Very Large Array at seven frequencies between 1.365 and $8.385 \mathrm{GHz}$. All of the observations are consistent with pure foreground Faraday rotation. We quantify the statistics of the magnetic-field fluctuations by deriving rotation measure structure functions, which we fit using models derived from theoretical power spectra. We quantify the errors due to sampling by making multiple twodimensional realizations of the best-fitting power spectrum. We also use depolarization measurements to estimate the minimum scale of the field variations. We then develop three-dimensional models with a gas density distribution derived from X-ray observations and a random magnetic field with this power spectrum. By comparing our simulations with the observed Faraday rotation images, we can determine the strength of the magnetic field and its dependence on density, as well as the outer scale of the magnetic turbulence. Results. The rotation measure and depolarization data are consistent with a broken power-law magnetic-field power spectrum, with a break at about $11 \mathrm{kpc}$ and slopes of 2.98 and 2.07 at smaller and larger scales respectively. The maximum and minimum scales of the fluctuations are $\approx 65$ and $\approx 0.2 \mathrm{kpc}$, respectively. The average magnetic field strength at the cluster centre is $3.5 \pm 1.2 \mu \mathrm{G}$, decreasing linearly with the gas density within $\approx 16 \mathrm{kpc}$ of the nucleus. At larger distances, the dependence of field on density appears to flatten, but this may be an effect of errors in the density model. The magnetic field is not energetically important.
\end{abstract}

Key words. magnetic fields - polarization - intergalactic medium - radio continuum: galaxies - X-rays: galaxies

\section{Introduction}

Magnetic fields in the hot plasma associated with groups and clusters of galaxies are poorly understood, but are thought to play a vital role in regulating thermal conduction (e.g. Balbus 2000; Bogdanovic et al. 2009) and influence the dynamics of cavities formed by radio jets (e.g. Dursi \& Pfrommer 2008; O'Neill et al. 2009). The existence of magnetic fields can be demonstrated in several different ways (e.g. Carilli \& Taylor 2002; Govoni \& Feretti 2004, and references therein). One of these is via the Faraday effect: rotation of the plane of linearly polarized radiation by a magnetized plasma. Synchrotron emission from radio sources (either behind or embedded within the group/cluster medium) can be used to probe the distribution of foreground Faraday rotation. These can be combined with X-ray observations (which provide the thermal gas density profile) to infer the strength and fluctuation properties of the magnetic field.

Faraday rotation studies of clusters have been carried out with both statistical samples of background radio sources (e.g. Lawler \& Dennison 1982; Vallée et al. 1986; Kim et al. 1990, 1991; Clarke et al. 2001) and individual radio sources within the clusters (e.g. Taylor \& Perley 1993; Feretti et al. 1995; Feretti et al. 1999a,b; Govoni et al. 2001; Eilek \& Owen 2002; Pollack et al. 2005; Govoni et al. 2006; Guidetti et al. 2008). The central magnetic field strengths deduced from these data are usually a few $\mu \mathrm{G}$, but can exceed $10 \mu \mathrm{G}$ in the inner regions of relaxed cool-core clusters (e.g. Taylor et al. 2002). The rotation measure (RM) distributions of radio galaxies in both interacting and relaxed clusters are generally patchy, indicating that cluster magnetic fields show a structure on scales $\$ 10 \mathrm{kpc}$.

Several studies of Abell clusters (Murgia et al. 2004; Govoni et al. 2006; Guidetti et al. 2008) have shown that detailed RM images of radio galaxies can be used to infer not only the strength of the cluster magnetic field, but also its power spectrum. The analysis of Vogt \& Enßlin $(2003,2005)$ suggests that the power spectrum has a power law form with the slope appropriate for Kolmogorov turbulence and that the auto-correlation length of the magnetic field fluctuations is a few kpc. The deduction of a Kolmogorov slope could be premature, however: there is a degeneracy between the slope and the outer scale, which is difficult to resolve with current Faraday rotation data (Murgia et al. 2004; Guidetti et al. 2008; Laing et al. 2008). Indeed, Murgia et al. (2004) pointed out that shallower magnetic field power spectra are possible if the magnetic field fluctuations have structure on scales of several tens of kpc. Recently, Guidetti et al. (2008) showed that a power-law power spectrum with a Kolmogorov slope, and an abrupt long-wavelength cutoff at $35 \mathrm{kpc}$ gave a very good fit to their Faraday rotation and 
depolarization data for the radio galaxies in A2382, although a shallower slope extending to longer wavelengths was not ruled out.

While most work until recently has been devoted to rich clusters of galaxies, little attention has been given in the literature to sparser environments, although similar physical processes are likely to be at work. Faraday-rotation fluctuations have previously been detected in galaxy groups (e.g. Perley et al. 1984; Feretti et al. 1999a), but without deriving in detail the geometry and structure of the magnetic field. The first detailed work on galaxy groups was done by Laing et al. (2008), who analysed the radio emission of $3 \mathrm{C} 31$.

They found that the three-dimensional magnetic-field power spectrum $\widehat{w}(f)$, defined in Sect. 6.1, can be described in terms of spatial frequency $f$ by a broken power-law $\widehat{w}(f) \propto D_{0} f^{-q}$ with $q=11 / 3^{1}$ for $f>0.062 \operatorname{arcsec}^{-1}$ (corresponding to a spatial scale of about $17 \mathrm{kpc}$ ) and $q=2.32$ at lower frequencies, although a power spectrum with a slope of 2.39 and an abrupt cut-off at high frequencies could not be ruled out. Their results are qualitatively similar to those for sources in Abell clusters.

Magnetic fields associated with galaxy groups deserve to be investigated in more detail, since their environments are more representative than those of rich clusters. Moreover, observations, analytical models and MHD simulations of galaxy clusters all suggest that the magnetic-field intensity should scale with the thermal gas density (e.g. Brunetti et al. 2001; Dolag 2006; Guidetti et al. 2008). A key question is whether the relation between magnetic field strength and density in galaxy groups is a continuation of this trend.

This paper presents a detailed analysis of Faraday rotation in 3C 449, a bright, extended radio source hosted by the central galaxy of a nearby group. With the aim of shedding new light on the environment around this source, we derive the statistical properties of the magnetic field from observations of Faraday rotation, following the method developed by Murgia et al. (2004). We use numerical simulations to predict the Faraday rotation for different strengths and power spectra of the magnetic field.

The paper is organized as follows. In Sect. 2 the general properties of the radio source under investigation are presented. Section 3 presents the radio images on which our analysis is based. In Sect. 4, we discuss the observed Faraday rotation distribution of 3C 449 and assess the contribution from our Galaxy. The observed depolarization and its relation to the RM properties are investigated in Sect. 5. Our two-dimensional analysis of the structure of the RM fluctuations and a three-dimensional model of the magnetic field consistent with these results are presented in Sects. 6 and 7, respectively. Section 8 summarizes our conclusions and briefly compares the Faraday-rotation properties of $3 \mathrm{C} 449$ with those of other sources.

Throughout this paper we assume a cosmology with $H_{0}=$ $71 \mathrm{~km} \mathrm{~s}^{-1} \mathrm{Mpc}^{-1}, \Omega_{\mathrm{m}}=0.3$, and $\Omega_{\Lambda}=0.7$, which implies that 1 arcsec corresponds to $0.342 \mathrm{kpc}$ at the distance of $3 \mathrm{C} 449$.

\section{The radio source $3 \mathrm{C} 449$ : general properties}

We image and model the Faraday rotation distribution across the giant Fanaroff-Riley Class I (FR I; Fanaroff \& Riley 1974) radio source $3 \mathrm{C} 449$, whose environment is very similar to that of 3C 31. The optical counterpart of 3C 449, UGC 12064, is a dumb-bell galaxy and is the most prominent member of the group of galaxies 2231.2+3732 (Zwicky \& Kowal 1968).

\footnotetext{
${ }^{1} q=11 / 3$ is the slope of the three-dimensional power spectrum for Kolmogorov turbulence.
}

The source is relatively nearby $(z=0.017085$, RC3.9, De Vaucouleurs et al. 1991) and quite extended, both in angular $\left(30^{\prime}\right)$ and linear size, so it is an ideal target for an analysis of the Faraday rotation distribution: detailed images can be constructed that can serve as the basis of an accurate study of magnetic field power spectra.

The source 3C 449 was one of the first radio galaxies studied in detail with the VLA $^{2}$ (Perley et al. 1979). High- and low-resolution radio data already exist and the source has been mapped at many frequencies. The radio emission of 3C 449 (Fig. 1) is elongated in the $\mathrm{N}-\mathrm{S}$ direction and is characterized by long, two-sided jets with a striking mirror symmetry close to the nucleus. The jets terminate in well-defined inner lobes, which fade into well polarized spurs, of which the southern one is more collimated. The spurs in turn expand to form diffuse outer lobes.

The brightness ratio of the radio jets is very nearly 1 , implying that they are close to the plane of the sky if they are intrinsically symmetrical and have relativistic flow velocities similar to those derived for other FR I jets (Perley et al. 1979; Feretti et al. 1999a; Laing \& Bridle 2002). We therefore assume that the jets lie exactly in the plane of the sky, which simplifies the geometry of the Faraday-rotating medium.

Hot gas associated with the galaxy was detected on both the group and the galactic scales by X-ray imaging (Hardcastle et al. 1998; Croston et al. 2003). These observations revealed deficits in the X-ray surface brightness at the positions of the outer radio lobes, suggesting interactions with the surrounding material. Figure 1 shows radio contours at $1.365 \mathrm{GHz}$ overlaid on the X-ray emission as observed by the XMM-Newton satellite (Croston et al. 2003). The X-ray radial surface brightness profile of 3C 449 derived from these data can be fitted with the sum of a point-source convolved with the instrumental response and a $\beta$ model (Cavaliere \& Fusco-Femiano 1976),

$n_{\mathrm{e}}(r)=n_{0}\left(1+r^{2} / r_{\mathrm{c}}^{2}\right)^{-\frac{3}{2} \beta}$,

where $r, r_{\mathrm{c}}$ and $n_{0}$ are the distance from the group X-ray centre, the group core radius, and the central electron density, respectively. Croston et al. (2008) found a best fitting model with $\beta=0.42 \pm 0.05, r_{\mathrm{c}}=57.1$ arcsec and $n_{0}=3.7 \times 10^{-3} \mathrm{~cm}^{-3}$. In our calculations, we assumed that the group gas density is described by the model of Croston et al. (2008). The X-ray depressions noted by Croston et al. (2003) are at distances larger than those at which we can measure linear polarization, and there is no direct evidence of smaller cavities close to the nucleus. We therefore neglect any departures from the spherically-symmetrical density model, noting that this approximation may become increasingly inaccurate where the source widens (i.e. in the inner lobes and spurs).

The source 3C 449 resembles 3C 31 in environment and in radio morphology: both sources are associated with the central members of groups of galaxies, and their redshifts are very similar. The nearest neighbours are at a projected distances of about $30 \mathrm{kpc}$ in both cases. Both radio sources have large angular extents, bending jets and long, narrow tails with low surface brightnesses and steep spectra, although 3C 31 appears much more distorted on large scales. There is one significant difference: the inner jets of $3 \mathrm{C} 31$ are thought to be inclined by $\approx 50^{\circ}$ to the line-of-sight (Laing \& Bridle 2002), whereas those in 3C 449 are likely to be close to the plane of the sky (Feretti et al. 1999a). We therefore expect that the magnetized foreground medium will be very similar in the two sources, but that the geometry

\footnotetext{
2 The very large array is a facility of the National Science Foundation, operated under cooperative agreement by Associated Universities, Inc.
} 


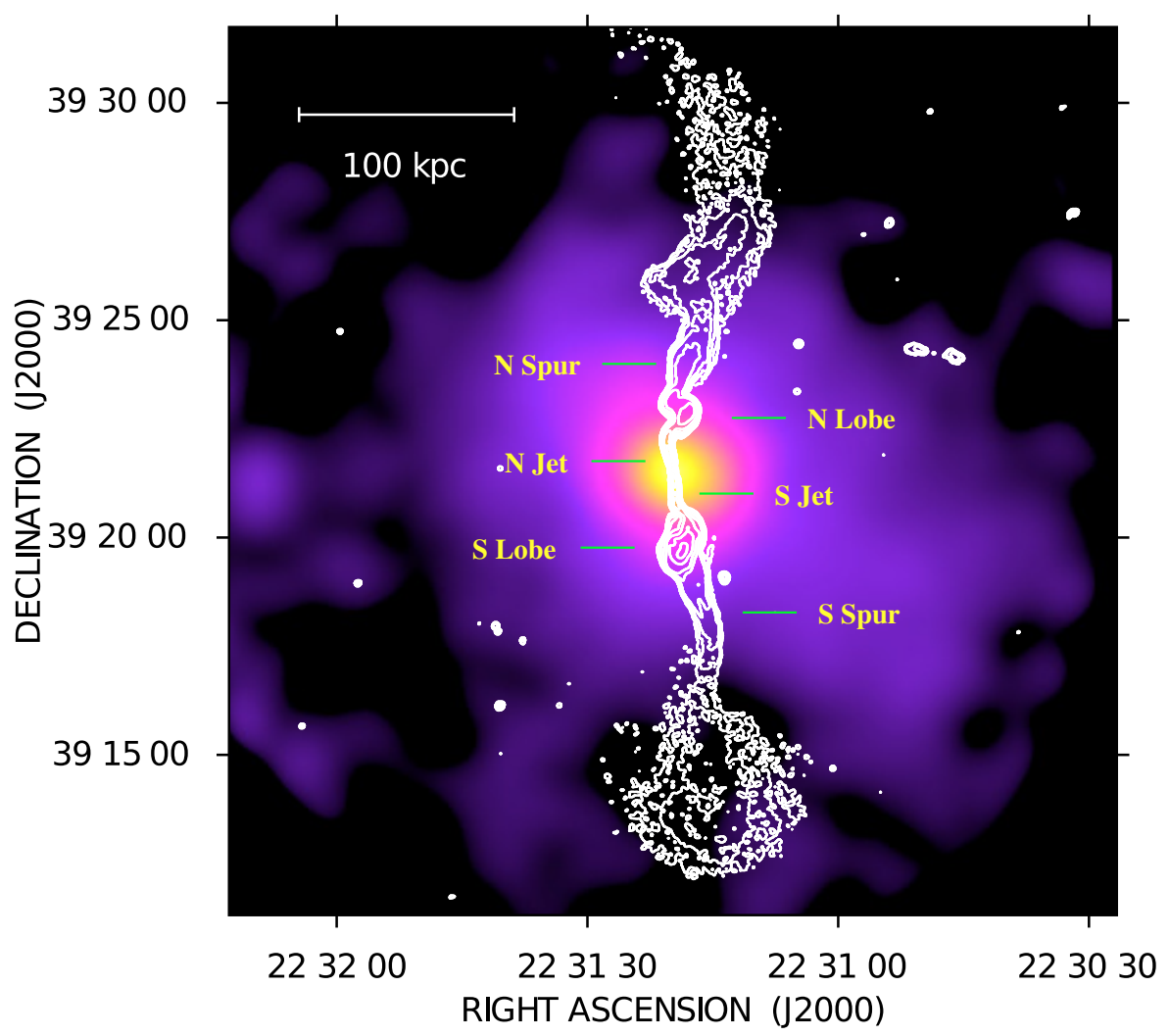

Fig. 1. Radio contours of $3 \mathrm{C} 449$ at $1.365 \mathrm{GHz}$ superposed on the XMM-Newton X-ray image (courtesy of J. Croston, Croston et al. 2003). The radio contours start at $3 \sigma_{I}$ and increase by factors of 2. The restoring beam is $5.5 \mathrm{arcsec}$ $F W H M$. The main regions of $3 \mathrm{C} 449$ discussed in the text are labelled. will be significantly different, leading to a much more symmetrical distribution of Faraday rotation in 3C 449 compared with that observed in 3C 31 by Laing et al. (2008).

\section{Total intensity and polarization properties}

The VLA observations and their reduction were presented by Feretti et al. (1999a). The high quality of these data make this source suited for a very detailed analysis of the statistics of the Faraday rotation.

We produced total intensity $(I)$ and polarization $(Q$ and $U$ ) images at frequencies in the range $1.365-8.385 \mathrm{GHz}$ from the combined, self-calibrated $u-v$ datasets described by Feretti et al. (1999a). The centre frequencies and bandwidths are listed in Table 1. Each frequency channel was imaged separately, except for those at 8.245 and $8.445 \mathrm{GHz}$, which were averaged. The analysis below confirms that these frequency-bandwidth combinations lead to negligible Faraday rotation across the channels, as already noted by Feretti et al. (1999a). All of the datasets were imaged with Gaussian tapering in the $u-v$ plane to give resolutions of $1.25 \operatorname{arcsec}$ and $5.5 \operatorname{arcsec} F W H M$, CLEANed and restored with circular Gaussian beams. The first angular resolution is the highest possible at all frequencies and provides good signal-to-noise for the radio emission within 150 arcsec $(\simeq 50 \mathrm{kpc})$ of the radio core (the well defined radio jets and the inner lobes), while minimizing beam depolarization. The lower resolution of 5.5 arcsec allows imaging of the extended emission as far as $300 \operatorname{arcsec}(\simeq 100 \mathrm{kpc})$ from the core at frequencies from $1.365-4.985 \mathrm{GHz}$ (the $8.385-\mathrm{GHz}$ dataset does not have adequate sensitivity to image the outer parts of the source). We can therefore study the structure of the magnetic field in the spur regions, which lie well outside the bulk of the X-ray emitting gas. Noise levels for both sets of images are given in Table 1. Note that the maximum scales of structure, which can be imaged reliably with the VLA at 8.4 and $5 \mathrm{GHz}$ are $\approx 180$ and $\approx 300$ arcsec, respectively (Ulvestad et al. 2009). For this reason, we only use the Stokes $I$ images for quantitative analysis within half these distances of the core. The $Q$ and $U$ images have much less structure on these large scales and are reliable to distances of \pm 150 arcsec at $8.4 \mathrm{GHz}$ and \pm 300 arcsec at $5 \mathrm{GHz}$, limited by sensitivity rather than systematic errors due to missing flux as in the case of the $I$ image.

Images of polarized intensity $P=\left(Q^{2}+U^{2}\right)^{1 / 2}$ (corrected for Ricean bias, following Wardle \& Kronberg 1974), fractional polarization $p=P / I$ and polarization angle $\Psi=(1 / 2) \arctan (U / Q)$ were derived from the $I, Q$, and $U$ images.

All of the polarization images $(P, p, \Psi)$ at a given frequency were blanked where the rms error in $\Psi>10^{\circ}$ at any frequency. We then calculated the scalar mean degree of polarization $\langle p\rangle$ for each frequency and resolution; the results are listed in Table 1 . The values of $\langle p\rangle$ are higher at $5.5 \mathrm{arcsec}$ resolution than at 1.25 arcsec because of the contribution of the extended and highly polarized emission, which is not seen at the higher resolution. At $1.25 \mathrm{arcsec}$, where the beam depolarization is minimized, the mean fractional polarization shows a steady increase from 1.365 to $4.685 \mathrm{GHz}$, where it reaches an average value of 0.32 and then remains roughly constant at higher frequencies, suggesting that the depolarization between 4.685 and $8.385 \mathrm{GHz}$ is insignificant.

\section{The Faraday rotation in $3 C 449$}

\subsection{Rotation measure images}

A magnetized, ionized medium rotates the plane of polarization of linearly polarized radiation passing through it as follows:

$\Delta \Psi=\Psi(\lambda)-\Psi_{0}=\mathrm{RM} \lambda^{2}$, 
Table 1. Parameters of the total intensity and polarization images.

\begin{tabular}{cccccccc}
\hline \hline$v$ & Bandwidth & \multicolumn{3}{c}{1.25 arcsec } & & \multicolumn{3}{c}{5.5 arcsec } & \\
$(\mathrm{GHz})$ & $(\mathrm{MHz})$ & $(\mathrm{mJy} / \mathrm{beam})$ & $\begin{array}{c}\sigma_{Q U} \\
(\mathrm{mJy} / \mathrm{beam})\end{array}$ & $\langle p\rangle$ & $\begin{array}{c}\sigma_{I} \\
(\mathrm{mJy} / \mathrm{beam})\end{array}$ & $\begin{array}{c}\sigma_{Q U} \\
(\mathrm{mJy} / \mathrm{beam})\end{array}$ & $\langle p\rangle$ \\
\hline 1.365 & 12.5 & 0.037 & 0.030 & 0.24 & 0.018 & 0.014 & 0.26 \\
1.445 & 12.5 & 0.021 & 0.020 & 0.25 & 0.020 & 0.011 & 0.27 \\
1.465 & 12.5 & 0.048 & 0.049 & 0.25 & 0.019 & 0.013 & 0.25 \\
1.485 & 12.5 & 0.035 & 0.027 & 0.21 & 0.014 & 0.010 & 0.26 \\
4.685 & 50.0 & 0.017 & 0.017 & 0.32 & 0.017 & 0.013 & 0.37 \\
4.985 & 50.0 & 0.018 & 0.017 & 0.33 & 0.017 & 0.016 & 0.39 \\
8.385 & 100.0 & 0.014 & 0.011 & 0.31 & 0.015 & 0.013 & - \\
\hline
\end{tabular}

Notes. Column 1: observation frequency. Column 2: bandwidth (note that the images at $8.385 \mathrm{GHz}$ are derived from the average of two frequency channels, both with bandwidths of $50 \mathrm{MHz}$, centred on 8.285 and $8.485 \mathrm{GHz})$; Cols. $3,4:$ rms noise levels in total intensity $\left(\sigma_{I}\right)$ and linear polarization $\left(\sigma_{Q U}\right.$, the average of $\sigma_{Q}$ and $\left.\sigma_{U}\right)$ at $1.25 \operatorname{arcsec} F W H M$ resolution; Col. 5: mean degree of polarization at 1.25 arcsec; Cols. 6, 7: rms noise levels for the 5.5 arcsec images; Col. 8: mean degree of polarization at 5.5 arcsec. We estimate that the uncertainty in the degree of polarization, which is dominated by systematic deconvolution errors on the $I$ images, is $\approx 0.02$ at each frequency.

where $\Psi(\lambda)$ is the position angle observed at a wavelength $\lambda$ and $\Psi_{0}$ is the intrinsic position angle. The rotation measure is related to the electron density $\left(n_{\mathrm{e}}\right)$, the magnetic field along the line-of-sight $\left(B_{\|}\right)$, and the path-length $(L)$ through the Faradayrotating medium according to

$\mathrm{RM}_{\left[\mathrm{rad} / \mathrm{m}^{2}\right]}=812 \int_{0}^{L_{[\mathrm{kpc}]}} n_{\mathrm{e}\left[\mathrm{cm}^{-3}\right]} B_{\|[\mu G]} \mathrm{d} l$.

Images of RM can be obtained for radio sources by fitting to the polarization angle as a function of $\lambda^{2}$, taking into account the well-known problem of $\mathrm{n} \pi$ ambiguities in the observed $\Psi$, as is done for example by the AIPS task RM. Removal of these ambiguities requires observations at many wavelengths well-spaced in $\lambda^{2}$.

We produced images of RM and its associated rms error with resolutions of $1.25 \operatorname{arcsec}$ and $5.5 \operatorname{arcsec}$ (Figs. 2a and b) using a version of the AIPS task RM modified by G. B. Taylor. The 1.25 arcsec-RM map was made by combining the maps of the polarization $\boldsymbol{E}$-vector $(\Psi)$ at all seven frequencies available to us, so our sampling of $\lambda^{2}$ is very good. The RM map was calculated with a weighted least-squares fit at pixels with polarization angle uncertainties $<10^{\circ}$ at all frequencies. It is essentially the same as the RM image of Feretti et al. (1999a), but with more stringent blanking. The average fitting error is $\simeq 1.4 \mathrm{rad} \mathrm{m}^{-2}$ and is almost constant over the whole RM image. The image of $\mathrm{RM}$ at 5.5 arcsec resolution was produced using the polarization position angles at the six frequencies between 1.365 and $4.985 \mathrm{GHz}$ (see Table 1) with the same blanking criterion as at higher resolution.

Patches with different size are apparent in the 1.25 arcsec resolution map, with fluctuations down to scales of a few $\mathrm{kpc}$. The $\mathrm{RM}$ values range from about $-220 \mathrm{rad} \mathrm{m}^{-2}$ up to $-90 \mathrm{rad} \mathrm{m}^{-2}$, dominated by the Galactic contribution (see Sect. 4.2). The $\mathrm{RM}$ distribution peaks at $-161.7 \mathrm{rad} \mathrm{m}^{-2}$, with a rms dispersion $\sigma_{\mathrm{RM}}=19.7 \mathrm{rad} \mathrm{m}^{-2}$. Note that we have not corrected the values of $\sigma_{\mathrm{RM}}$ for the fitting error $\sigma_{\mathrm{RM}_{\text {fit }}}$. A first order correction would be $\sigma_{\mathrm{RM}_{\text {true }}}=\left(\sigma_{\mathrm{RM}}^{2}-\sigma_{\mathrm{RM}_{\mathrm{fit}}}^{2}\right)^{1 / 2}$. Given the low value for $\sigma_{\mathrm{RM}_{\mathrm{fit}}}$, the effect of this correction would be very small.

As was noted by Feretti et al. (1999a), the RM distribution in the inner jets is highly symmetric about the core with $\mathrm{RM} \simeq-197 \mathrm{rad} \mathrm{m}^{-2}$ at distances $\lesssim 15$ arcsec. The symmetry of the RM distribution in the jets is broken at larger distances from the core: while the RM structure in the southern jet is homogeneous, with values around $-130 \mathrm{rad} \mathrm{m}^{-2}$, fluctuations on scales of $\simeq 10 \operatorname{arcsec}(\simeq 3 \mathrm{kpc})$ around $\mathrm{a}\langle\mathrm{RM}\rangle$ of $-160 \mathrm{rad} \mathrm{m}^{-2}$ are present in the northern jet. In both lobes, we observe similar patchy RM structures with mean values $\langle\mathrm{RM}\rangle \simeq-164 \mathrm{rad} \mathrm{m}^{-2}$ and $\sigma_{\mathrm{RM}} \simeq 16 \mathrm{rad} \mathrm{m}^{-2}$.

At 5.5 arcsec resolution, more extended polarized regions of 3C 449 can be mapped with good sampling in $\lambda^{2}$. The average fitting error is $\simeq 1.0 \mathrm{rad} \mathrm{m}^{-2}$. Both spurs are characterized by $\langle\mathrm{RM}\rangle \simeq-160 \mathrm{rad} \mathrm{m}^{-2}$, with $\sigma_{\mathrm{RM}}=15$ and $10 \mathrm{rad} \mathrm{m}^{-2}$ in the north and south, respectively. The overall mean and $\mathrm{rms}$ for the $5.5 \mathrm{arcsec}$ image, $\langle\mathrm{RM}\rangle=-160.7 \mathrm{rad} \mathrm{m}^{-2}$ and $\sigma_{\mathrm{RM}}=18.9 \mathrm{rad} \mathrm{m}^{-2}$, are very close to those determined at higher resolution and are consistent with the integrated value of $-162 \pm 1 \mathrm{rad} \mathrm{m}^{-2}$ derived by Simard-Normandin et al. (1981).

It was demonstrated by Feretti et al. (1999a) that the polarization position angles at 1.25 arcsec resolution accurately follow the relation $\Delta \Psi \propto \lambda^{2}$ over a wide range of rotation. We find the same effect at lower resolution: plots of $\boldsymbol{E}$-vector position angle $\Psi$ against $\lambda^{2}$ at representative points of the 5.5 arcsecRM image are shown in Fig. 3. As at the higher resolution, there are no significant deviations from the relation $\Delta \Psi \propto \lambda^{2}$ over a range of rotation $\Delta \Psi$ of $600^{\circ}$, confirming that a foreground magnetized medium is responsible for the majority of the Faraday rotation and extending this result to regions of lower surface brightness.

In Figs. 2c and d, we show profiles of $\sigma_{\mathrm{RM}}$ for both low and high resolution RM images. The 1.25 arcsec profile was obtained by averaging over boxes with lengths ranging from 9 to $13 \mathrm{kpc}$ along the radio axis; for the 5.5 arcsec profile we used boxes with a fixed length of $9 \mathrm{kpc}$ (these sizes were chosen to give an adequate number of independent points per box). The boxes extend far enough perpendicular to the source axis to include all unblanked pixels. In both plots, there is clear evidence for a decrease in the observed $\sigma_{\mathrm{RM}}$ towards the periphery of the source, the value dropping from $\simeq 30 \mathrm{rad} \mathrm{m}^{-2}$ close to the nucleus to $\simeq 10 \mathrm{rad} \mathrm{m}^{-2}$ at $50 \mathrm{kpc}$. This is qualitatively as expected for foreground Faraday rotation by a medium whose density (and presumably also magnetic field strength) decreases with radius. The symmetry of the $\sigma_{\text {RM }}$ profiles is consistent with our assumption that the radio source lies in the plane of the sky.

\subsection{The Galactic Faraday rotation}

For the purpose of this work, 3C 449 has an unfortunate lineof-sight within our Galaxy. Firstly, the source is located at $l=95.4^{\circ}, b=-15.9^{\circ}$ in Galactic coordinates, where the Galactic magnetic field is known to be aligned almost along the 

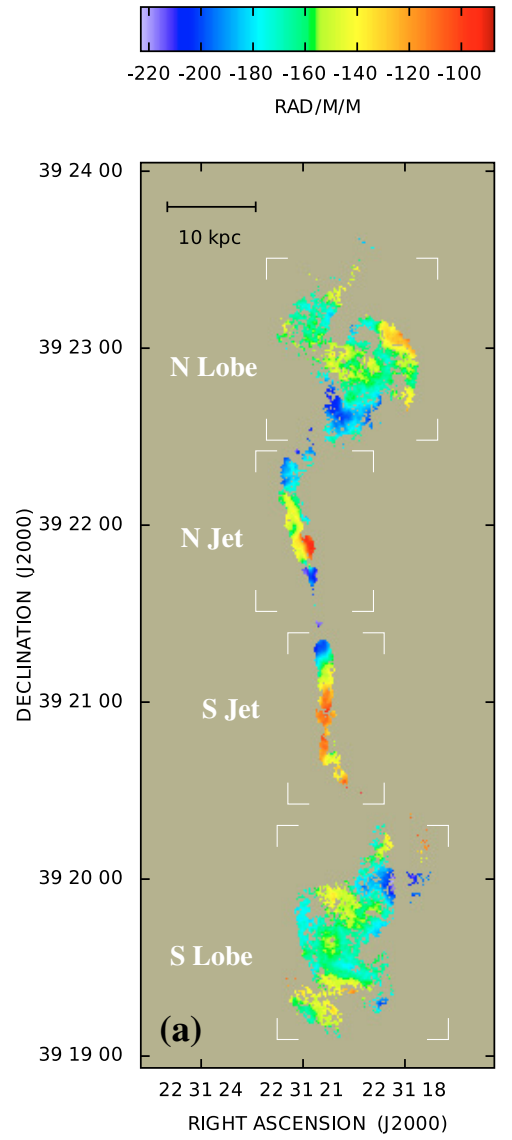
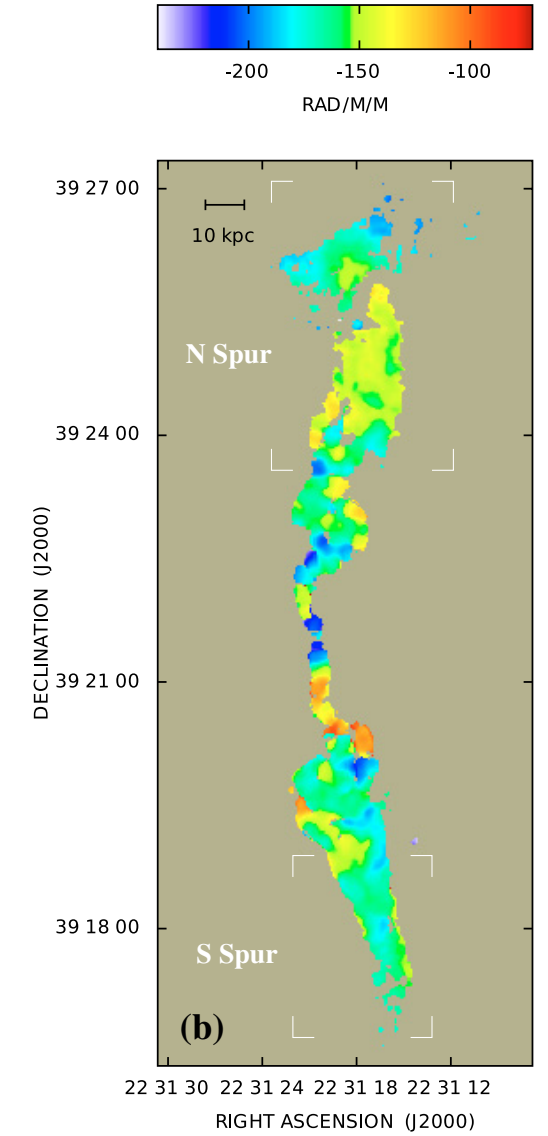
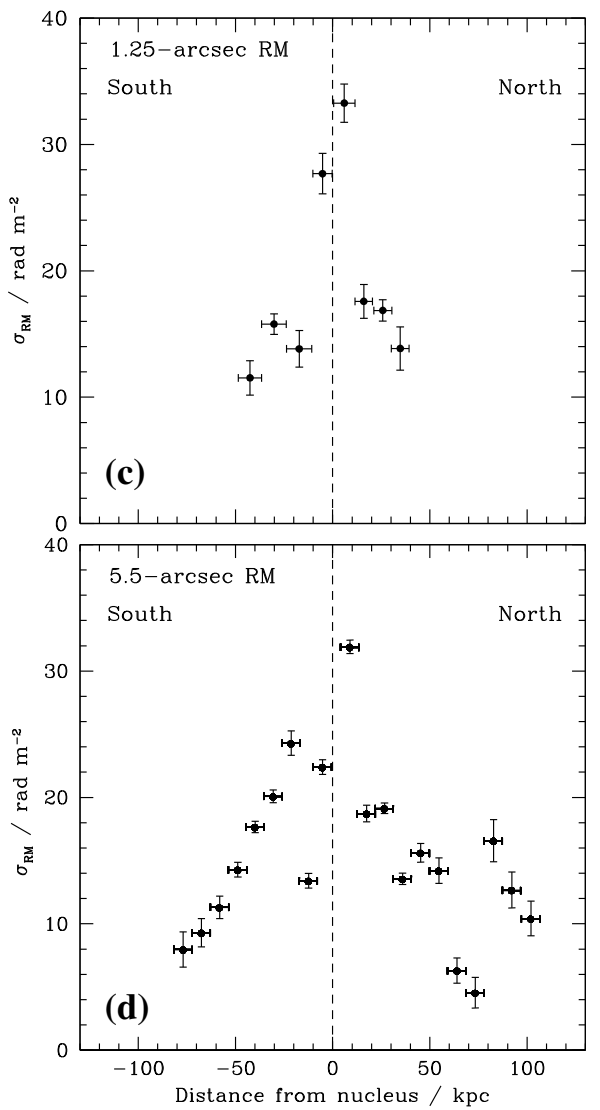

Fig. 2. a) Image of the rotation measure of $3 \mathrm{C} 449$ at a resolution of $1.25 \operatorname{arcsec} F W H M$, computed at the seven frequencies between 1.365 and $8.385 \mathrm{GHz}$. b) Image of the rotation measure of $3 \mathrm{C} 449$ at a resolution of $5.5 \operatorname{arcsec} F W H M$, computed at the six frequencies between 1.365 and $4.985 \mathrm{GHz}$. In both of the RM images, the sub-regions used for the two-dimensional analysis of Sect. 6 are labelled. c) and d) profiles of $\sigma_{\mathrm{RM}}$ as a function of the projected distance from the radio source centre. The points represent the values of $\sigma_{\mathrm{RM}}$ evaluated in boxes as described in the text. The horizontal and vertical bars represent the bin widths and the rms on the mean expected from fitting errors, respectively. Positive distances are in the direction of the north jet and the vertical dashed lines show the position of the nucleus.

line-of-sight. Secondly, there is evidence from radio and optical imaging for a diffuse, ionized Galactic feature in front of $3 \mathrm{C} 449$, perhaps associated with the nearby HII region S126 (Andernach et al. 1992). Estimates of the Galactic foreground $\mathrm{RM}$ at the position of 3C 449 from observations of other radio sources are uncertain: Andernach et al. (1992) found a mean value of $-212 \mathrm{rad} \mathrm{m}^{-2}$ for six nearby sources, but the spherical harmonic models of Dineen \& Coles (2005), which are derived by fitting to the RM values of large numbers of extragalactic sources, predict $-135 \mathrm{rad} \mathrm{m}^{-2}$. Nevertheless, it is clear that the bulk of the mean RM of 3C 449 must be Galactic.

In order to investigate the magnetized plasma local to 3C 449, we need to constrain the value and possible spatial variation of this Galactic contribution. The profiles of $\sigma_{\mathrm{RM}}$ (Fig. 2) show that the small-scale fluctuations of RM drop rapidly with distance from the nucleus. We might therefore expect the Galactic contribution to dominate on the largest scales. At low resolution, we could determine the RM accurately out to $\approx 100 \mathrm{kpc}$ from the core. This is roughly 5 core radii for the $\mathrm{X}$-ray emission and therefore well outside the bulk of the intragroup gas.

In order to estimate the Galactic RM contribution, we averaged the 5.5-arcsec RM image in boxes of length $20 \mathrm{kpc}$ along the radio axis (the box size has been increased from that of Fig. 2 to improve the display of large-scale variations). The profile of $\langle\mathrm{RM}\rangle$ against the distance from the radio core is shown in Fig. 4.
The large deviations from the mean in the innermost two bins are associated with the maximum in $\sigma_{\mathrm{RM}}$ and are almost certainly due to the intra-group medium. The dispersion in $\langle\mathrm{RM}\rangle$ is quite small in the south and the value of $\langle\mathrm{RM}\rangle=-160.7 \mathrm{rad} \mathrm{m}^{-2}$ for the whole source is very close to that of the outer south jet. There are significant fluctuations in the north, however. Given their rather small scale $(\sim 300$ arcsec $)$, it is most likely that these arise in the local environment of 3C 449, and we include them in the statistical analysis given below.

There is some evidence for linear gradients in Galactic RM on arcminute scales: Laing et al. (2006) found a gradient of $0.025 \mathrm{rad} \mathrm{m}^{-2} \operatorname{arcsec}^{-1}$ along the jets of the radio galaxy $\operatorname{NGC} 315\left(l=124.6^{\circ}, b=-32.5^{\circ}\right)$. They argued that this gradient is almost certainly Galactic in origin, since the amplitude of the linear variation exceeds that of the small-scale fluctuations associated with NGC 315. In order to check the effect of a large-scale Galactic RM gradient on our results, we computed an unweighted least-squares fit of a function $\langle\mathrm{RM}\rangle=\mathrm{RM}_{0}+a x$, where $a$ and $\mathrm{RM}_{0}$ are constant and $x$ is measured along the radio axis. The two innermost bins in Fig. 4 were excluded from the fit. Our best estimate for the gradient is very small: $a=0.0054 \mathrm{rad} \mathrm{m}^{-2} \operatorname{arcsec}^{-1}$. We have verified that subtraction of this gradient has a negligible effect on the structure-function analysis given in Sect. 7.3.

We therefore adopt a constant value of $-160.7 \mathrm{rad} \mathrm{m}^{-2}$ as the Galactic contribution. 

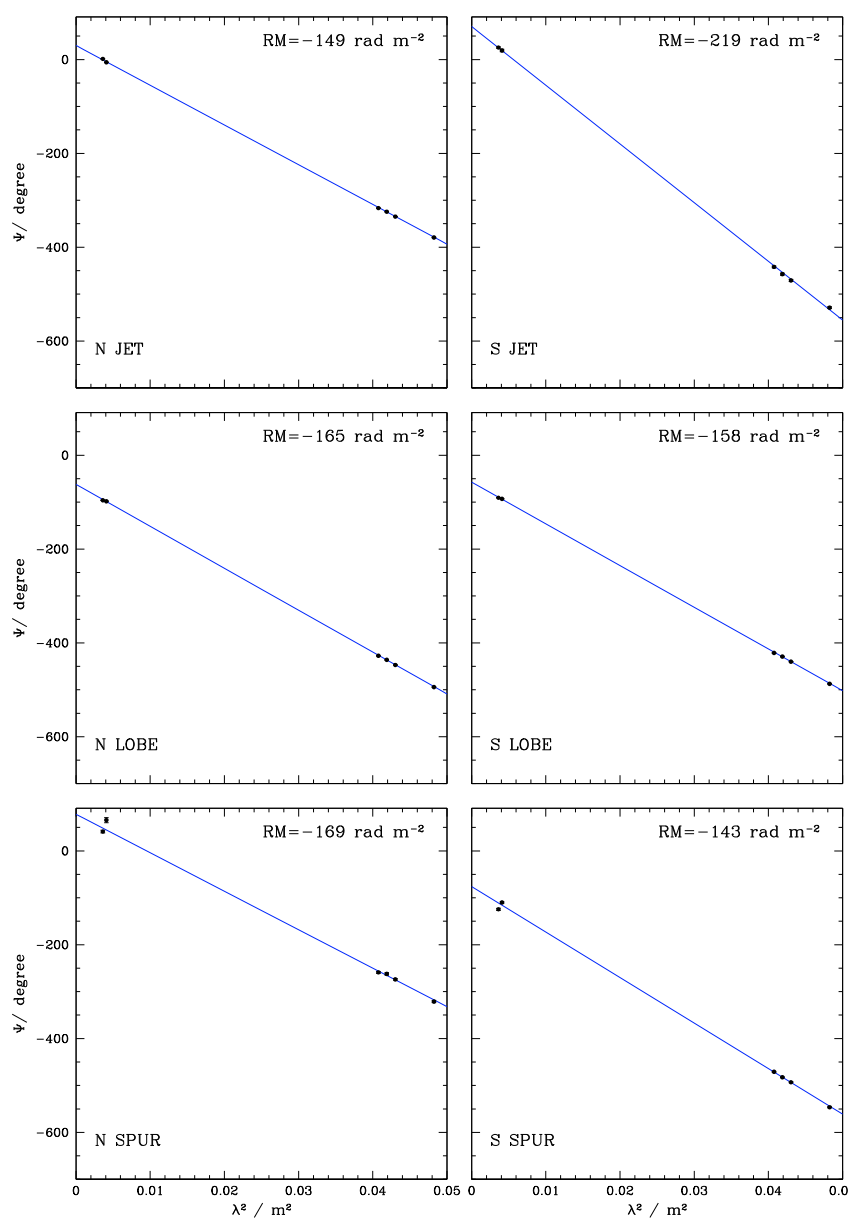

Fig. 3. Plots of $\boldsymbol{E}$-vector position angle $\Psi$ against $\lambda^{2}$ at representative points of the 5.5-arcsec RM map. Fits to the relation $\Psi(\lambda)=\Psi_{0}+\mathrm{RM} \lambda^{2}$ are shown. The values of RM are given in the individual panels.

\section{Depolarization}

Faraday rotation generally leads to a decrease of the degree of polarization with increasing wavelength, or depolarization (DP). We define $\mathrm{DP}_{\lambda_{2}}^{\lambda_{1}}=p\left(\lambda_{1}\right) / p\left(\lambda_{2}\right)$, where $p(\lambda)$ is the degree of polarization at a given wavelength $\lambda$. We adopt the conventional usage, in which higher depolarization corresponds to a lower value of DP.

Laing (1984) summarized the interpretation of polarization data. Faraday depolarization of radio emission from radio sources can occur in three principal ways:

1. thermal plasma is mixed with the synchrotron emitting material (internal depolarization);

2. there are fluctuations of the foreground Faraday rotation across the beam (beam depolarization); and

3. the polarization angle varies across the finite band of the receiving system (bandwidth depolarization).

We first estimated the bandwidth effects on the polarized emission of 3C 449 using the RM measurements from Sect. 4.1. In the worst case (the highest absolute RM value of $-240 \mathrm{rad} \mathrm{m}^{-2}$ ) at the lowest frequency of $1.365 \mathrm{GHz}$ ) the rotation across the band is $\approx 10^{\circ}$. This results in a depolarization of $1.7 \%$, negligible compared with errors due to noise.

If $\lambda^{2}$ rotation is observed over a position-angle range $\gg 90^{\circ}$, then a foreground screen must be responsible for the bulk of the observed RM. In that case, depolarization can still result from

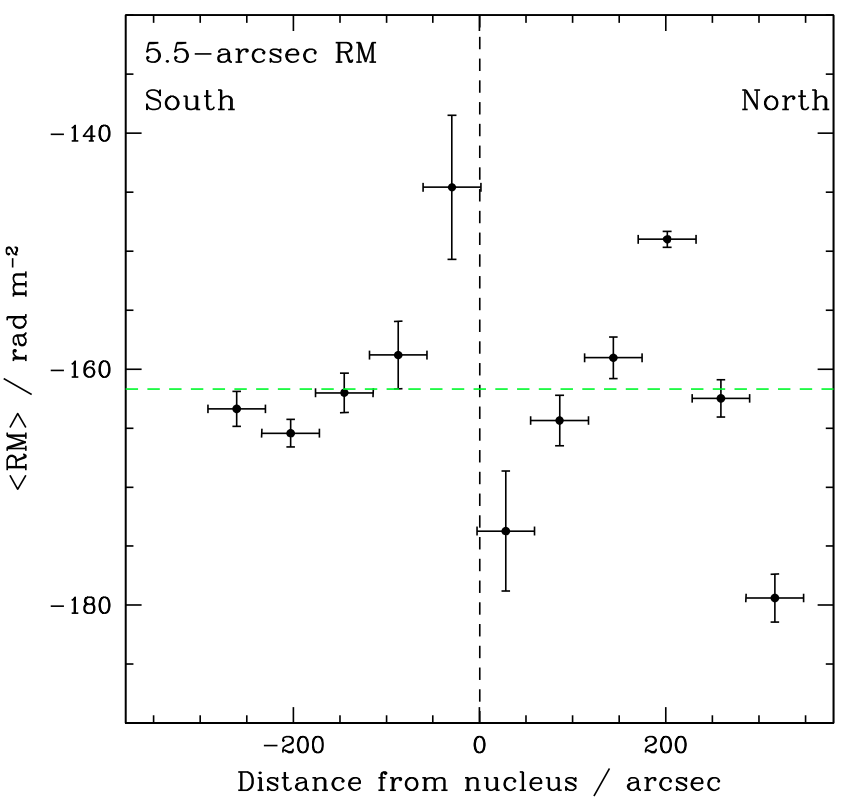

Fig. 4. Profile of RM averaged over boxes of length $20 \mathrm{kpc}$ along the radio axis for the 5.5 arcsec image. The horizontal bars represent the bin width. The vertical bars are the errors on the mean calculated from the dispersion in the boxes, the contribution from the fitting error is negligible and is not taken into account. Positive distances are in the direction of the north jet. The black vertical dashed line indicates the position of the nucleus; the green dashed line shows our adopted mean value for the Galactic RM.

unresolved inhomogeneities of thermal density or magnetic field in the surrounding medium. Our analysis of the depolarization of 3C 449 is based on the approach of Laing et al. (2008). It is still possible to observe $\lambda^{2}$ rotation over a wide range of polarization angle from a foreground Faraday screen with a small gradient of RM across the beam. In this case, the wavelength dependence of the depolarization is expected to follow the Burn law (Burn 1966)

$p(\lambda)=p(0) \exp \left(-k \lambda^{4}\right)$,

where $p(0)$ is the intrinsic value of the degree of polarization and $k=2|\nabla R M|^{2} \sigma^{2}$, with $F W H M=2 \sigma(2 \ln 2)^{1 / 2}$. Since $k \propto|\nabla \mathrm{RM}|^{2}$, Eq. (4) clearly illustrates that higher RM gradients across the beam generate higher $k$ values and hence higher depolarization. The variation of $p$ with wavelength can potentially be used to estimate fluctuations of RM across the beam, which are below the resolution limit. We can determine the intrinsic polarization $p(0)$ and the proportionality constant $k$ by a linear fit to the logarithm of the observed fractional polarization as a function of $\lambda^{4}$.

We made images of $k$ at both standard resolutions by weighted least-squares fitting to the fractional polarization maps, using the FARADAY code by M. Murgia. The same frequencies were used as for the RM images: $8.385-1.365 \mathrm{GHz}$ and $4.985-1.365 \mathrm{GHz}$ at 1.25 and 5.5 arcsec resolution, respectively. By simulating the error distributions for $p$, we established that the mean values of $k$ were biased significantly at low signal-tonoise (cf. Laing et al. 2008), so only data with $p>4 \sigma_{\mathrm{p}}$ at each frequency are included in the fits. We estimate that any bias is negligible compared with the fitting error. We also derived profiles of $k$ with the same sets of boxes as for the $\sigma_{\mathrm{RM}}$ profiles in Fig. 2.

The $1.25 \operatorname{arcsec}$ resolution $k$ map is shown in Fig. 5a, together with the profile of the $k$ values (Fig. 5c). The fit to a $\lambda^{4}$ law 

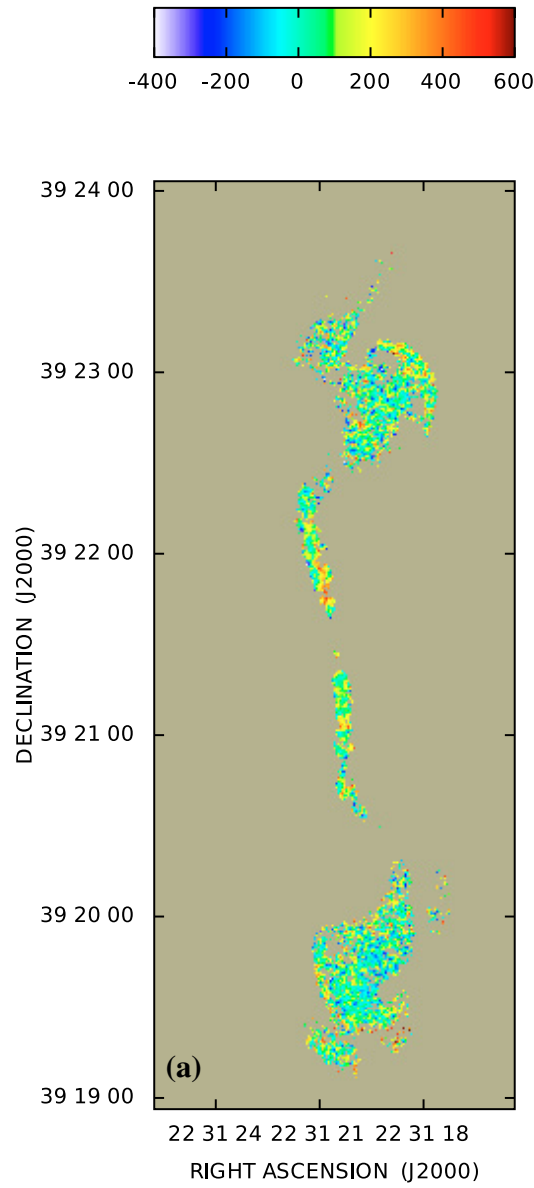

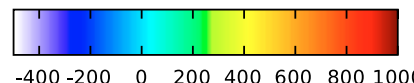

$-400-200 \quad 0 \quad 2004006008001000$

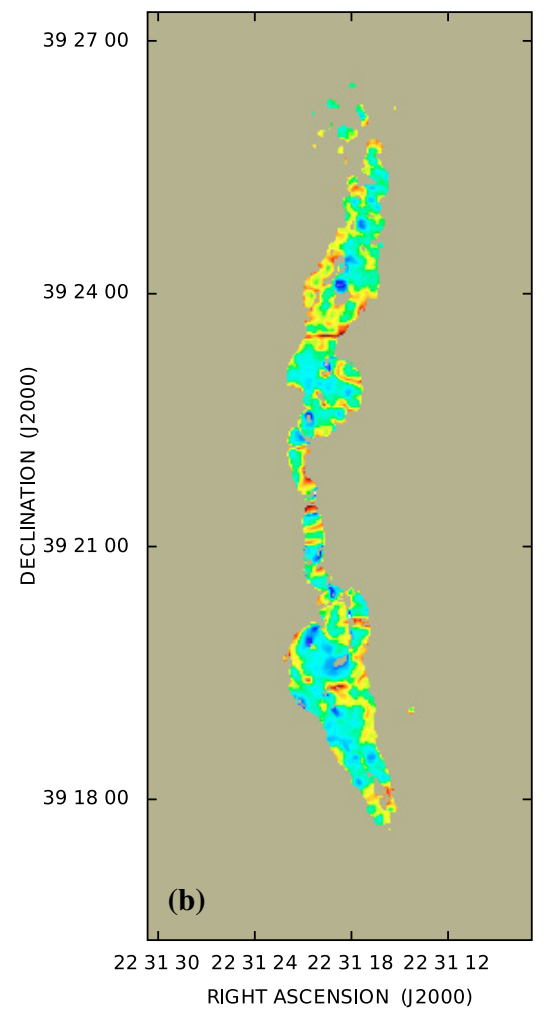

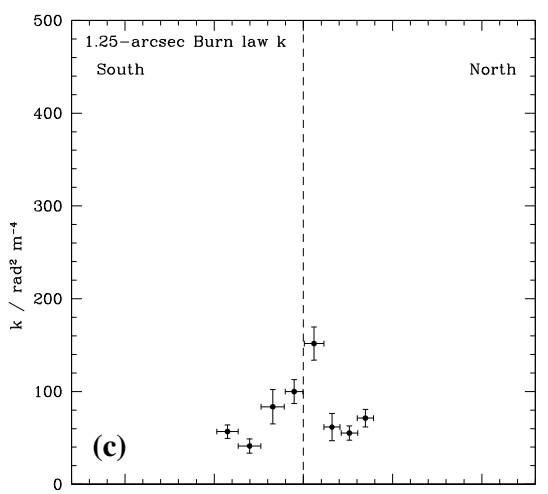

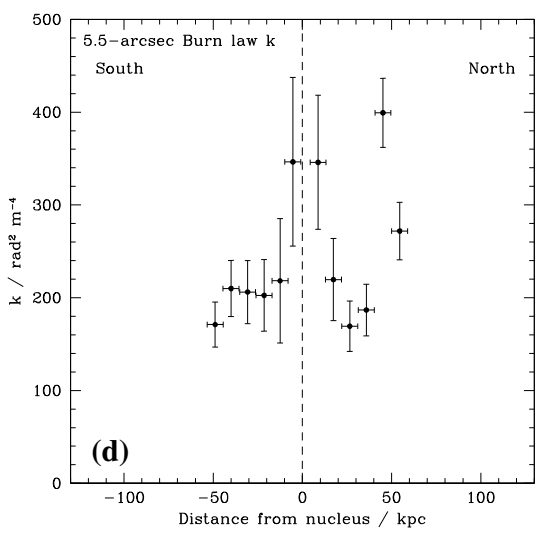

Fig. 5. a) Image of the Burn law $k$ in $\operatorname{rad}^{2} \mathrm{~m}^{-4}$ computed from a fit to the relation $p(\lambda)=p(0) \exp \left(-k \lambda^{4}\right)$ for seven frequencies between 1.365 and $8.385 \mathrm{GHz}$. b) as a), but the angular resolution is $5.5 \operatorname{arcsec} F W H M$, and the $k$ image have been computed computed from the fit to the six frequencies between 1.365 and $4.985 \mathrm{GHz}$. c) and d) profiles for $k$ as functions of the projected distance from the radio source centre (boxes as in Fig. 2). The horizontal and vertical bars represent the bin widths and the error on the mean, respectively. Positive distances are in the direction of the north jet, and the vertical dashed lines show the position of the nucleus.

is very good everywhere: examples of fits at selected pixels in the jets and lobes are shown in in Fig. 6. The symmetry observed in the $\sigma_{\mathrm{RM}}$ profiles is also seen in the $1.25 \operatorname{arcsec} k$ image (Fig. 5): the mean values of $k$ are $\simeq 50 \mathrm{rad}^{2} \mathrm{~m}^{-4}$ for both lobes, 107 and $82 \mathrm{rad}^{2} \mathrm{~m}^{-4}$ for the northern and southern jet, respectively. The region with the highest depolarization is in the northern jet, very close to the core and along the west side. The integrated value of $k$ at this resolution is $\simeq 56 \mathrm{rad}^{2} \mathrm{~m}^{-4}$, corresponding to a mean depolarization $D P_{3 \mathrm{~cm}}^{20 \mathrm{~cm}} \simeq 0.87$.

The image and profile of $k$ at 5.5-arcsec resolution are shown in Figs. $5 b$ and d. The fit to a $\lambda^{4}$ law is in general good and examples are shown in in Fig. 6. As mentioned earlier, the maximum scale of structure imaged accurately in total intensity at $5 \mathrm{GHz}$ is $\sim 300$ arcsec $(100 \mathrm{kpc})$ and there are likely to be significant systematic errors in the degree of polarization on larger scales. We therefore show the profile only for the inner $\pm 50 \mathrm{kpc}$. Over this range, the $k$ profiles are quite symmetrical, as at higher resolution. Note also that the small regions of very high $k$ at the edge of the northern and southern spurs in the map shown in Fig. 5 are likely to be spurious.

The mean values of $k$ are $\simeq 184 \mathrm{rad}^{2} \mathrm{~m}^{-4}$ and $178 \mathrm{rad}^{2} \mathrm{~m}^{-4}$ for the northern and southern lobes, respectively; and $\simeq 238$ and $174 \mathrm{rad}^{2} \mathrm{~m}^{-4}$ in the northern and in the southern spurs. The integrated value of $k$ is $\simeq 194 \mathrm{rad}^{2} \mathrm{~m}^{-4}$, corresponding to a depolarization $D P_{6 \mathrm{~cm}}^{20 \mathrm{~cm}} \simeq 0.64$.

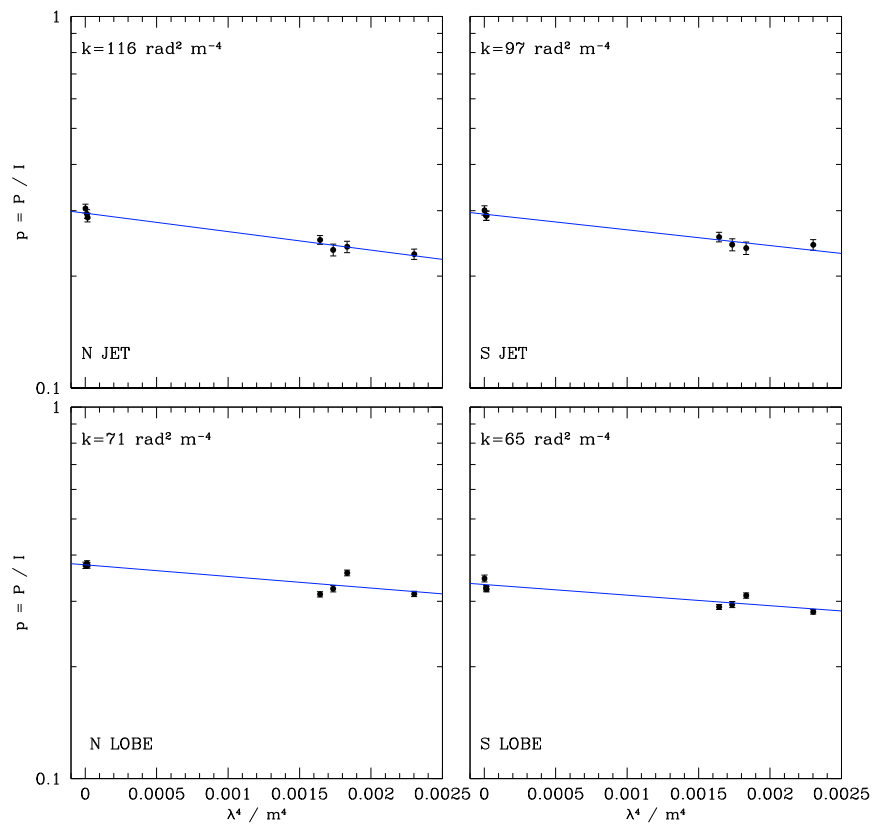

Fig. 6. Plots of degree of polarization, $p$ (log scale) against $\lambda^{4}$ for representative points at 1.25 -arcsec resolution. Burn law fits (Eq. (4)) are also plotted. The values of $k$ are quoted in the individual panels. 


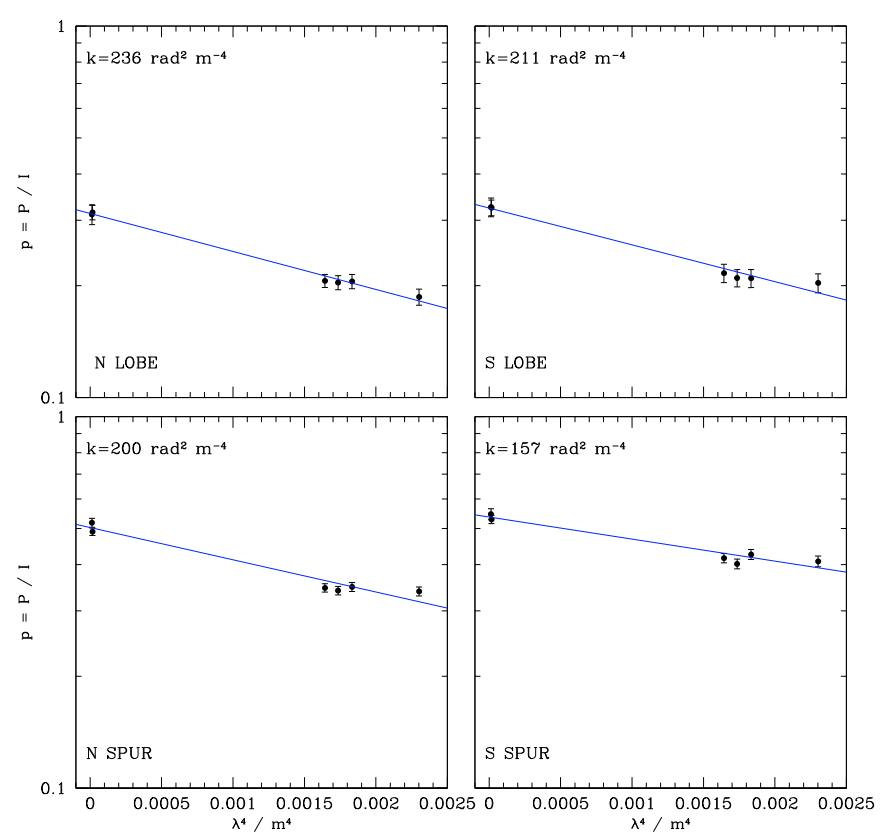

Fig. 7. Plots of degree of polarization, $p$ (log scale) against $\lambda^{4}$ for representative points at 5.5-arcsec resolution. Burn law fits (Eq. (4)) are also plotted. The values of $k$ are quoted in the individual panels.

To summarize, we observe depolarization between $20 \mathrm{~cm}$ and $3 \mathrm{~cm}$. Since we measure lower values of $k$ at $1.25 \operatorname{arcsec}$ than 5.5 arcsec, there is less depolarization at high resolution, as expected for beam depolarization. The highest depolarization is observed in a region of the northern jet, close to the radio core and associated with a steep RM gradient. Depolarization is significantly higher close to the nucleus, which is consistent with the higher path length through the group gas observed in $\mathrm{X}$-rays. Aside from this global variation, we found no evidence for a detailed correlation of depolarization with source structure. Depolarization and RM data are therefore both consistent with a foreground Faraday screen. We show in Sect. 6.2 that the residual depolarization at 1.25 -arcsec resolution can be produced by RM fluctuations on scales smaller than the beamwidth, but higher-resolution observations are needed to establish this conclusively.

\section{Two dimensional analysis}

\subsection{General considerations}

In order to interpret the fluctuations of the magnetic field responsible for the observed RM and depolarization of 3C 449, we first discuss the statistics of the RM fluctuations in two dimensions. We use the notation of Laing et al. (2008), in which $\boldsymbol{f}=\left(f_{x}, f_{y}, f_{z}\right)$ is a vector in the spatial frequency domain, corresponding to the position vector $\boldsymbol{r}=(x, y, z)$. We take the $z$-axis to be along the line-of-sight, so that the vector $\boldsymbol{r}_{\perp}=(x, y)$ is in the plane of the sky and $\boldsymbol{f}_{\perp}=\left(f_{x}, f_{y}\right)$ is the corresponding spatial frequency vector. Our goal is to estimate the RM power spectrum $\widehat{C}\left(\boldsymbol{f}_{\perp}\right)$, where $\widehat{C}\left(\boldsymbol{f}_{\perp}\right) \mathrm{d} f_{x} \mathrm{~d} f_{y}$ is the power in the area $\mathrm{d} f_{x} \mathrm{~d} f_{y}$ and in turn to derive the three-dimensional magnetic-field power spectrum $\widehat{w}(\boldsymbol{f})$, defined so that $\widehat{w}(\boldsymbol{f}) \mathrm{d} f_{x} \mathrm{~d} f_{y} \mathrm{~d} f_{z}$ is the power in a volume $\mathrm{d} f_{x} \mathrm{~d} f_{y} \mathrm{~d} f_{z}$ of frequency space.

The relation between the magnetic field statistics and the observed RM distribution is in general quite complicated, depending on the fluctuations in the thermal gas density, the geometry of the source and the surrounding medium, and the effects of incomplete sampling. In order to derive the magnetic-field power spectrum, we make the following simplifying assumptions, as in Guidetti et al. (2008) and Laing et al. (2008).

1. The observed Faraday rotation is due entirely to a foreground ionized medium (in agreement with our results in Sects. 4 and 5);

2. the magnetic field is an isotropic, Gaussian random variable, and can therefore be characterized by a power spectrum $\widehat{w}(f)$, which is a function only of the scalar frequency $f$;

3 . the form of the magnetic field power spectrum is independent of position;

4. the magnetic field is distributed throughout the Faradayrotating medium, whose density is a smooth, spherically symmetric function;

5. the amplitude of $\widehat{w}(f)$ is spatially variable, but is a function only of the thermal electron density.

These assumptions guarantee that the spatial distribution of the magnetic field can be described entirely by its power spectrum $\widehat{w}(f)$ and that for a medium of constant depth and density, the power spectra of magnetic field and RM are proportional (Enßlin \& Vogt 2003).

If the fluctuations are isotropic, the RM power spectrum $\widehat{C}\left(f_{\perp}\right)$ is the Hankel transform of the autocorrelation function $C\left(r_{\perp}\right)$, defined as

$C\left(r_{\perp}\right)=\left\langle\operatorname{RM}\left(r_{\perp}+r_{\perp}^{\prime}\right) \operatorname{RM}\left(r_{\perp}^{\prime}\right)\right\rangle$

where $\boldsymbol{r}_{\perp}$ and $\boldsymbol{r}_{\perp}$ are vectors in the plane of the sky and \langle\rangle is an average over $\boldsymbol{r}_{\perp}^{\prime}$.

In an ideal case, it would be possible to derive the RM power spectrum and consequently that of the magnetic field directly from $C\left(r_{\perp}\right)$. In reality, the observations are affected first by the effects of convolution with the beam, which modify the spatial statistics of RM, and secondly, by the limited size and irregular shape of the sampling region for 3C 449, which results in a complicated window function (Enßlin \& Vogt 2003) and limits the accuracy with which the zero-level can be determined. In Sect. 4.2, we showed that the Galactic contribution to the $3 \mathrm{C} 449 \mathrm{RM}$ is substantial and argued that a constant value of $-160.7 \mathrm{rad} \mathrm{m}^{-2}$ is the best estimate for its value. Fluctuations in the Galactic magnetic field on scales comparable with the size of the radio sources could be present; conversely, the local environment of the source might make a significant contribution to the mean RM. Both of these possibilities lead to difficulties in the use of the autocorrelation function.

Laing et al. (2008) demonstrated a procedure that takes into account the convolution effects and minimises the effects of uncertainties in the zero-level. In particular, they showed that

1. in the short-wavelength limit (meaning that changes in Faraday rotation across the beam are adequately represented as a linear gradient), the measured RM distribution is closely approximated by the convolution of the true RM distribution with the observing beam;

2. the structure function is a powerful and reliable statistical tool to quantify the two dimensional fluctuations of RM, given that it is independent of the zero level and structure on scales larger than the area under investigation.

The structure function is defined by

$S\left(r_{\perp}\right)=\left\langle\left[\mathrm{RM}\left(\boldsymbol{r}_{\perp}+\boldsymbol{r}_{\perp}^{\prime}\right)-\mathrm{RM}\left(\boldsymbol{r}_{\perp}^{\prime}\right)\right]^{2}\right\rangle$ 
(Simonetti et al. 1984; Minter \& Spangler 1996). It is related to the autocorrelation function $C\left(r_{\perp}\right)$ for a sufficiently large averaging region by $S\left(r_{\perp}\right)=2\left[C\left(r_{\perp}\right)-C(0)\right]$.

Laing et al. (2008) also derived the effects of convolution with the observing beam on the observed structure function. For the special case of a power-law power spectrum (their Eq. (B2)), they showed that the observed structure function after convolution can be heavily modified even at separations up to a few times the $F W H M$ of the observing beam. This effect must be taken into account when comparing observed and predicted structure functions. Laing et al. (2008) and Guidetti et al. (2008) also showed that numerical simulations of depolarization provide complementary information on RM fluctuations on scales smaller than the beam.

Following the approach of Laing et al. (2008), we initially used the RM structure function to determine the form of $\widehat{w}(f)$ (Sect. 6.2), while for its normalization (determined by global variations of density and magnetic field strength), we made use of three-dimensional simulations (Sect. 7).

\subsection{Structure functions}

We calculated the structure function for discrete regions of 3C 449, over which we expected the spatial variations of thermal gas density, rms magnetic field strength and path length to be reasonably small. For each of these regions, we first made unweighted fits of model structure functions derived from power spectra with simple, parameterized functional forms, accounting for convolution with the observing beam. We then generated multiple realizations of a Gaussian, isotropic, random RM field, with the best-fitting power spectrum on the observed grids, again taking into account the effects of the convolving beam. Finally, we made a weighted fit using the dispersion of the synthetic structure functions as estimates of the statistical errors for the $o b$ served structure functions, which are impossible to quantify analytically (Laing et al. 2008). These errors, which result from incomplete sampling, are much larger than those due to noise, but depend only weakly on the precise form of the underlying power spectrum. Our measure of the goodness of fit is $\chi^{2}$, summed over a range of separations from $r_{\perp}=F W H M$ to roughly half of the size of the region: there is no information in the structure function for scales smaller than the beam, and the upper limit is set by sampling. The errors are, of course, much higher at the large spatial scales, which are less well sampled. Note, however, that estimates of the structure function from neighbouring bins are not statistically independent, so it is not straightforward to define the effective number of degrees of freedom.

We selected six regions for the structure-function analysis, as shown in Fig. 2. These are symmetrically placed about the nucleus, consistent with the orientation of the radio jets close to the plane of the sky. For the north and south jets, we derived the structure functions only at 1.25 -arcsec resolution, as the low-resolution RM image shows no additional structure and has poorer sampling. For the north and south lobes, we computed the structure functions at both resolutions over identical areas and compared them. The agreement is very good, and the lowresolution RM images do not sample significantly larger spatial scales, so we show only the 1.25 -arcsec results. Finally, we used the 5.5-arcsec RM images to compute the structure functions for the north and south spurs, which are not detected at the higher resolution.

The structure function has a positive bias given by $2 \sigma_{\text {noise}}^{2}$, where $\sigma_{\text {noise }}$ is the uncorrelated random noise in the RM image (Simonetti et al. 1984). The mean noise of the 1.25 and 5.5-arcsec RM maps is $<1 \mathrm{rad} \mathrm{m}^{2}$ and is essentially uncorrelated on scales larger than the beam. For each region we therefore subtracted $2 \sigma_{\text {noise }}^{2}$ from the structure functions, although this correction is always small. The noise-corrected structure functions are shown in Fig. 8.

The individual observed structure functions have approximately power-law forms. Given that the structure function for a power-law power spectrum with no frequency limits is itself a power law (Minter \& Spangler 1996; Laing et al. 2008), we first tried to fit the observed data with a RM power spectrum of the form

$\widehat{C}\left(f_{\perp}\right) \propto f_{\perp}^{-q}$

over an infinite frequency range. This last assumption allows us to use the analytical solution of the structure function, including convolution (Laing et al. 2008), and therefore to avoid numerical integration.

The fits were quite good, but systematically gave slightly too much power on small spatial scales and over-predicted the depolarization. We therefore fit a cut-off power law (CPL) power spectrum

$$
\begin{aligned}
\widehat{C}\left(f_{\perp}\right) & =0 & & f_{\perp}<f_{\min } \\
& =C_{0} f_{\perp}^{-q} & & f_{\perp} \leq f_{\max } \\
& =0 & & f_{\perp}>f_{\max } .
\end{aligned}
$$

Initially, we consider values of $f_{\text {min }}$ sufficiently small that their effects on the structure functions over the observed range of separations are negligible. The free parameters of the fit in this case are the slope, $q$, the cut-off spatial frequency $f_{\max }$ and the normalization of the power spectrum, $C_{0}$. In Table 2 , we give the best-fitting parameters for CPL fits to all of the individual regions. The fitted model structure functions are plotted in Figs. $8 \mathrm{a}-\mathrm{f}$, together with error bars derived from multiple realizations of the power spectrum as in Laing et al. (2008).

In order to constrain RM structure on spatial scales below the beamwidth, we estimated the depolarization expected from the best power spectrum for each of the regions with 1.25-arcsec RM images, following the approach of Laing et al. (2008). To do this, we made multiple realizations of RM images on an $8192^{2}$ grid with fine spatial sampling. We then derived the $Q$ and $U$ images at our observing frequencies, convolved to the appropriate resolution and compared the predicted and observed mean degrees of polarization. These values are given in Table 4 . The uncertainties in the expected $\langle k\rangle$ in Table 4 represent statistical errors determined from multiple realizations of RM images with the same set of power spectrum parameters. The predicted and observed values are in excellent agreement. A constant value of $f_{\max }=1.67 \mathrm{arcsec}^{-1}$ predicts very similar values, also listed in Table 4. We have not compared the depolarization data at 5.5 -arcsec resolution in the spurs because of limited coverage of large spatial scales in the $I$ images (Sect. 3), which is likely to introduce systematic errors at 4.6 and $5.0 \mathrm{GHz}$.

We performed a joint fit of the CPL power spectra, minimizing the $\chi^{2}$ summed over all six sub-regions, giving equal weight to each and allowing the normalizations to vary independently. In this case the free parameters of the fit are the six normalizations (one for each sub-region), the slope, and the maximum spatial frequency. The joint best-fitting single power-law power spectrum has $q=2.68$. A single power law slope does not give a good fit to all of the regions simultaneously, however. It is clear from Fig. 8 and Table 2 that there is a flattening in the slope of the observed structure functions on the largest scales (which are sampled primarily by the spurs). In order to fit all of the data 

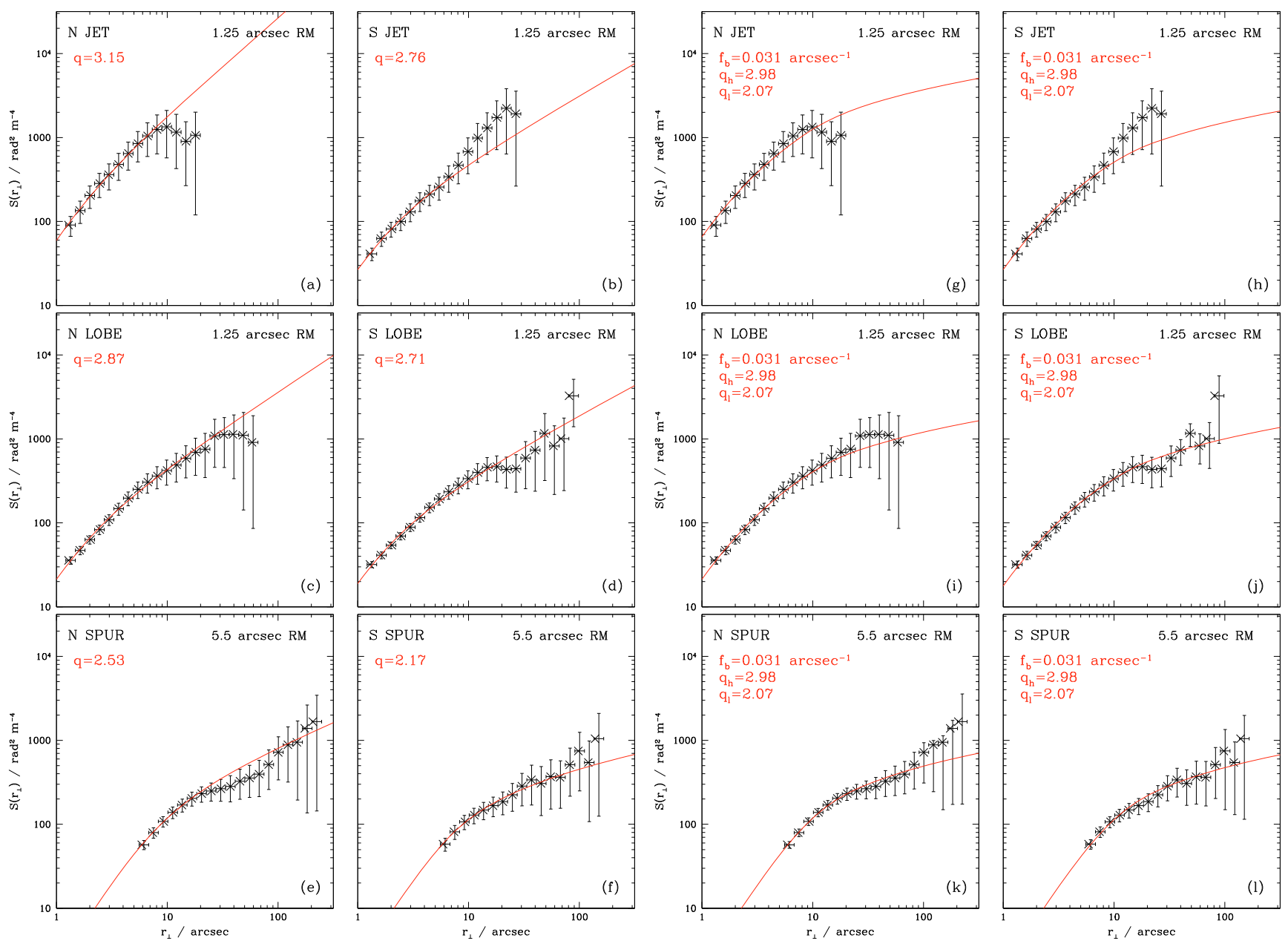

Fig. 8. a)-f) Plots of the RM structure functions for the sub-regions showed in Fig. 2. The horizontal bars represent the bin widths and the crosses the centroids for data included in the bins. The red lines are the predictions for the CPL power spectra described in the text, including the effects of the convolving beam. The vertical error bars are the rms variations for the structure functions derived using a CPL power spectrum with the quoted value of $q$ on the observed grid of points for each sub-region. g)-l) as a)-f), but using a BPL power spectra with fixed slopes and break frequency, but variable normalization.

Table 2. CPL power spectrum parameters for the six individual subregions of $3 \mathrm{C} 449$.

\begin{tabular}{ccccccccc}
\hline \hline Region & $F W H M$ & \multicolumn{1}{c}{ CPL } \\
& $\begin{array}{c}F \text { (arcsec }) \\
\text { best fit }\end{array}$ & \multicolumn{2}{c}{ min slope } & \multicolumn{2}{c}{ max slope } \\
& & $q$ & $f_{\max }$ & $q^{-}$ & $f_{\max }$ & $q^{+}$ & $f_{\max }$ \\
\hline N SPUR & 5.50 & 2.53 & 1.96 & 1.58 & 0.23 & 3.44 & $\infty$ \\
N LOBE & 1.25 & 2.87 & 1.60 & 2.31 & 0.55 & 3.35 & $\infty$ \\
N JET & 1.25 & 3.15 & 1.21 & 2.29 & 0.30 & 4.27 & $\infty$ \\
S JET & 1.25 & 2.76 & 1.95 & 2.02 & 0.3 & 3.69 & $\infty$ \\
S LOBE & 1.25 & 2.71 & 1.68 & 2.36 & 0.65 & 3.05 & $\infty$ \\
S SPUR & 5.50 & 2.17 & 1.53 & 0.20 & 0.12 & 3.95 & 0.12 \\
\hline
\end{tabular}

Notes. Lower and upper limits are quoted at $\sim 90 \%$ confidence.

accurately with a single functional form for the power spectrum, we adopt a broken power law form (BPL) for the RM power spectrum:

$$
\begin{aligned}
& \widehat{C}\left(f_{\perp}\right)=0 \\
& f_{\perp}<f_{\min } \\
& =D_{0} f_{\mathrm{b}}^{\left(q_{1}-q_{\mathrm{h}}\right)} f_{\perp}^{-q_{1}} \\
& f_{b} \geq f_{\perp} \\
& =D_{0} f_{\perp}^{-q_{\mathrm{h}}} \quad f_{\text {max }} \geq f_{\perp}>f_{b} \\
& =0 \quad f_{\perp}>f_{\text {max }} \text {. }
\end{aligned}
$$

We performed a BPL joint fit in the same way as for the CPL power spectra. In this case the free parameters of the fit are the six normalizations, $D_{0}$, one for each sub-region, the high and low-frequency slopes, $q_{\mathrm{h}}$ and $q_{\mathrm{l}}$, and the break and maximum spatial frequencies $f_{\mathrm{b}}$ and $f_{\max }$. We found best fitting parameters of $q_{1}=2.07, q_{\mathrm{h}}=2.98, f_{\mathrm{b}}=0.031 \operatorname{arcsec}^{-1}$. As noted earlier, we also fixed $f_{\text {max }}=1.67 \operatorname{arcsec}^{-1}$ to ensure consistency with the observed depolarizations at 1.25 -arcsec resolution. The corresponding structure functions are plotted in Figs. $8 \mathrm{~g}-1$ and the normalizations for the individual regions are given in Table 4. As for the CPL fits, the errors bars are derived from the rms scatter of the structure functions of multiple convolved RM realizations.

It is evident from Fig. 8 that the structure functions corresponding to the BPL power spectrum, which gives less power on large spatial scales, agree much better with the data. The joint BPL fit has a $\chi^{2}$ of 17.7 , compared with 33.5 for the joint CPL fit (the former has only two extra parameters), which confirms this result.

We have so far ignored the effects of any outer scale of the magnetic-field fluctuations. This is justified because the structure functions for the spurs continue to rise at the largest observed separations, indicating that the outer scale must be $\gtrsim 10 \operatorname{arcsec}(\simeq 30 \mathrm{kpc})$. The model structure functions fit to the observations assume that the outer scale is infinite and the 
Table 3. Best-fitting parameters for the joint CPL and BPL fits to all six sub-regions of 3C 449.

\begin{tabular}{|c|c|c|c|c|c|c|c|c|c|c|}
\hline & \multicolumn{4}{|c|}{ Best fit } & & \multicolumn{3}{|c|}{ Min slope } & \multicolumn{2}{|c|}{ Max slope } \\
\hline & $q$ & $f_{\max }$ & & $\chi^{2}$ & $q^{-}$ & $f_{\max }$ & & $q^{+}$ & $f_{\max }$ & \\
\hline \multirow[t]{3}{*}{ joint CPL } & 2.68 & 1.67 & & 33.5 & 2.55 & 1.30 & & 2.81 & 2.00 & \\
\hline & \multicolumn{4}{|c|}{ Best fit } & & \multicolumn{3}{|c|}{ Min slope } & \multicolumn{2}{|c|}{ Max slope } \\
\hline & $q_{l}$ & $f_{\mathrm{b}}$ & $q_{h}$ & $x^{2}$ & $q_{l}^{-}$ & $f_{\mathrm{b}}$ & $q_{h}^{-}$ & $q_{l}^{+}$ & $f_{\mathrm{b}}$ & $q_{l}^{+}$ \\
\hline joint BPL & 2.07 & 0.031 & 2.97 & 17.7 & 1.99 & 0.044 & 2.91 & 2.17 & 0.021 & 3.09 \\
\hline
\end{tabular}

Notes. Lower and upper limits are quoted at $\sim 90 \%$ confidence. The values of $q$ and $f_{\max }$ for the joint CPL fit and $q_{h}, q_{l}$ and $f_{\mathrm{b}}$ for the joint BPL fit are the same for all sub-regions, while the normalizations are varied to minimize the overall $\chi^{2}$. In the joint BPL fit, the maximum frequency is fixed at $f_{\max }=1.67 \operatorname{arcsec}^{-1}$.

Table 4. Normalizations and expected depolarization for the individual CPL, joint CPL and joint BPL fit parameters at 1.25 arcsec.

\begin{tabular}{|c|c|c|c|c|c|c|c|c|}
\hline \multirow[t]{2}{*}{ Region } & \multirow{2}{*}{$\begin{array}{c}\text { Observed }\langle k\rangle \\
\left(\operatorname{rad}^{2} \mathrm{~m}^{-4}\right)\end{array}$} & \multicolumn{3}{|c|}{ CPL } & \multicolumn{2}{|c|}{ JOINT CPL } & \multicolumn{2}{|c|}{ JOINT BPL } \\
\hline & & $C_{0}$ & $\begin{array}{c}f_{\max } \\
\left(\operatorname{arcsec}^{-1}\right)\end{array}$ & $\begin{array}{c}\langle k\rangle \\
\left(\operatorname{rad}^{2} \mathrm{~m}^{-4}\right)\end{array}$ & $C_{0}$ & $\begin{array}{c}\langle k\rangle \\
\left(\operatorname{rad}^{2} \mathrm{~m}^{-4}\right)\end{array}$ & & $\begin{array}{c}\langle k\rangle \\
\left(\operatorname{rad}^{2} \mathrm{~m}^{-4}\right)\end{array}$ \\
\hline N LOBE & $61 \pm 6$ & 0.96 & 1.60 & $63 \pm 3$ & 1.52 & $66 \pm 3$ & 1.91 & $52 \pm 4$ \\
\hline N JET & $106 \pm 12$ & 1.34 & 1.21 & $106 \pm 5$ & 4.76 & $110 \pm 5$ & 0.5 & $109 \pm 5$ \\
\hline S JET & $91 \pm 11$ & 1.50 & 1.95 & $70 \pm 5$ & 1.94 & $73 \pm 5$ & 1.52 & $65 \pm 4$ \\
\hline S LOBE & $50 \pm 5$ & 1.18 & 1.68 & $53 \pm 2$ & 1.28 & $50 \pm 2$ & 2.20 & $45 \pm 3$ \\
\hline
\end{tabular}

Notes. Column 1: region; Col. 2: observed Burn law $\langle k\rangle$. Columns 3-5: normalization constant $C_{0}$, fitted maximum spatial frequency $f_{\max }$ for the best CPL power spectrum of each region and the predicted $\langle k\rangle$ for each power spectrum. Columns 6,7 as Cols. 3 and 5 but for the joint fit to each CPL power spectrum; Cols. 8 and 9 as Cols. 3, and 5 but for the joint BPL power spectrum. For both the joint CPL and BPL fits, the maximum frequency is fixed at $f_{\max }=1.67 \operatorname{arcsec}^{-1}$. In calculating each value of $\langle k\rangle$ only data with $p>4 \sigma_{p}$ are included.

realizations are generated on sufficiently large grids in Fourier space that the effects of the implicit outer scale are negligible over the range of scales we sampled. We use structure-function data for the entire source to determine an approximate value for the outer scale in Sect. 7.3.

We now adopt the BPL power spectrum with these parameters and investigate the spatial variations of the RM fluctuation amplitude using three-dimensional simulations.

\section{Three-dimensional analysis}

\subsection{Models}

We used the software package FARADAY (Murgia et al. 2004) to compare the observed RM with simulated images derived from three-dimensional multi-scale magnetic-field models. Given a field model and the density distribution of the thermal gas, FARADAY calculates an RM image by integrating Eq. (3) numerically. As in Sect. 6, we modelled the fluctuations of RM on the assumption that the magnetic field responsible for the foreground rotation is an isotropic, Gaussian random variable and therefore characterized entirely by its power spectrum. Each point in a cube in Fourier space was first assigned components of the magnetic vector potential. The amplitudes were selected from a Rayleigh distribution of unit variance, and the phases were random in $[0,2 \pi]$. The amplitudes were then multiplied by the square root of the power spectrum of the vector potential, which is simply related to that of the magnetic field. The corresponding components of the magnetic field along the lineof-sight were then calculated and transformed to real space. This procedure ensured that the magnetic field was divergence-free. The field components in real space were then multiplied by the model density distribution and integrated along the line of sight to give a synthetic RM image at the full resolution of the simulation, which was then convolved to the observing resolution.
For 3C 449, we assumed that the source is in a plane perpendicular to the line- of-sight, which passes through the group centre and simulated the field and density structure using a $2048^{3}$ cube with a real-space pixel size of $0.1 \mathrm{kpc}$. We used the best-fitting BPL power spectrum found in Sect. 6.2, but with a spatially-variable normalization, as described below (Sect. 7.2), and a low-frequency cut-off $f_{\min }$, corresponding to a maximum scale of the magnetic field fluctuations ${ }^{3}, \Lambda_{\max }\left(=f_{\min }^{-1}\right)$. The power spectrum of Eq. (9) was then set to 0 for $f<f_{\min }$. We fixed the minimum scale of the fluctuations $\Lambda_{\min }=0.2 \mathrm{kpc}$. This is equivalent to the value $f_{\max }=1.67 \operatorname{arcsec}^{-1}$ found in Sect. 6.2 and also consistent with the requirement that the minimum scale can be no larger than twice the pixel size for adequate sampling.

We made multiple synthetic RM images at resolutions of 1.25 and 5.5 arcsec over the fields of view of the observations for each combination of parameters. In order to estimate the spatial variation of the magnetic-field strength, we first made a set of simulations with a large, fixed value of $\Lambda_{\max }$ and compared the predicted and observed profiles of $\sigma_{\mathrm{RM}}$ (Sect. 7.2). We then fixed the radial variation of the field at its best-fitting form and estimated the value of $\Lambda_{\max }$ with a structure-function analysis for the whole source (Sect. 7.3).

\subsection{Magnetic field strength and radial profile}

In order to estimate the radial variation of field strength, we first fixed the value of the outer scale to be $\Lambda_{\max }=205 \mathrm{kpc}$, the longest allowed by our simulation grid. Our approach was to make a large number of simulations for each combination of field strength and radial profile and to compare the predicted

\footnotetext{
${ }^{3}$ Here we refer to the scale length $\Lambda$ as a complete wavelength, i.e. $\Lambda=1 / f$. This differs by a factor of 2 from the definition in Guidetti et al. (2008), where $\Lambda$ is the reversal scale of the magnetic field, so $\Lambda=1 / 2 f$.
} 
Table 5. Summary of magnetic field power spectrum and density scaling parameters.

\begin{tabular}{cc}
\hline \hline & BPL power spectrum \\
\hline$q_{1}=2.07$ & low-frequency slope \\
$q_{\mathrm{h}}=2.98$ & high-frequency slope \\
$f_{b}=0.031 \operatorname{arcsec}^{-1}$ & break frequency $\left(\Lambda_{\mathrm{b}}=1 / f_{b}=11 \mathrm{kpc}\right)$ \\
$f_{\max }=1.67 \operatorname{arcsec}^{-1}$ & maximum frequency $\left(\Lambda_{\min }=1 / f_{\max }=0.2 \mathrm{kpc}\right)$ \\
$f_{\min }$ fitted & minimum frequency $\left(\Lambda_{\max }=1 / f_{\min }\right)$ \\
\hline & Scaling of the magnetic field \\
\hline$B_{0}$ fitted & Average magnetic field at group centre \\
$\eta$ fitted & Magnetic field exponent of the radial profile: $\langle B\rangle(r)=B_{1} 1860\left[\frac{n_{\mathrm{e}}(r)}{n_{0}}\right]^{\eta}$ \\
\hline
\end{tabular}

and observed values of $\sigma_{\mathrm{RM}}$ evaluated over the boxes used in Sect. 4.1 (Fig. 2). We used $\chi^{2}$ summed over the boxes as a measure of the goodness of fit. This procedure is independent of the precise value of the outer scale, provided that it is much larger than the averaging boxes. We express our results in terms of $\chi_{\text {red }}^{2}$, which is the value of $\chi^{2}$ divided by the number of degrees of freedom.

We initially tried a radial field-strength variation of the form

$\left\langle B^{2}(r)\right\rangle^{1 / 2}=B_{0}\left[\frac{n_{\mathrm{e}}(r)}{n_{0}}\right]^{\eta}$

as used by Guidetti et al. (2008) and Laing et al. (2008). Here, $B_{0}$ is the rms magnetic field strength at the group centre and $n_{\mathrm{e}}(r)$ is the thermal electron gas density, assumed to follow the $\beta$-model profile derived by Croston et al. (2008; see Sect. 2). This functional form is consistent with other observations, analytical models and numerical simulations. In particular, $\eta=2 / 3$ corresponds to flux-freezing and $\eta=1 / 2$ to equipartition between thermal and magnetic energy. Dolag et al. (2001) and Dolag (2006) found $\eta \approx 1$ from the correlation between the observed rms RM and X-ray surface brightness in galaxy groups and clusters and showed that this is consistent with the results of MHD simulations.

We produced simulated RM images for each combination of $B_{0}$ and $\eta$ in the ranges $0.5-10 \mu \mathrm{G}$ in steps of $0.1 \mu \mathrm{G}$ and $0-2$ in steps of 0.01 , respectively. We then derived the synthetic $\sigma_{\mathrm{RM}}$ profiles and, by comparing them with the observed one, calculated the unweighted $\chi^{2}$. We repeated this procedure 35 times at each angular resolution, noting the $\left(B_{0}, \eta\right)$ pair that gave the lowest $\chi^{2}$ in each case. These values are plotted in Fig. 9. As in earlier work (Murgia et al. 2004; Guidetti et al. 2008; Laing et al. 2008), we found a degeneracy between the values of $B_{0}$ and $\eta$ in the sense that the fitted values are positively correlated, but there are clear minima in $\chi^{2}$ at both resolutions. We therefore adopted the mean values of $B_{0}$ and $\eta$, weighted by $1 / \chi^{2}$, as the best overall estimates. These are also plotted in Fig. 9 as blue crosses. Although the central magnetic field strengths derived for the two RM images are consistent at the $1 \sigma$ level $\left(B_{0}=2.8 \pm 0.5 \mu \mathrm{G}\right.$ and $B_{0}=4.1 \pm 1.2 \mu \mathrm{G}$ at 5.5 and 1.25 -arcsec resolution, respectively), the values of $\eta$ are not. The best-fitting values are $\eta=0.0 \pm 0.1$ at $5.5 \operatorname{arcsec} F W H M$ and $\eta=0.8 \pm 0.4$ at $1.25 \operatorname{arcsec} F W H M$.

We next produced 35 RM simulations at each angular resolution by fixing $B_{0}$ and $\eta$ at their best values for that resolution. This allowed us to calculate weighted $\chi^{2}$ 's for the $\sigma_{\mathrm{RM}}$ profiles, evaluating the errors for each box by summing in quadrature the rms due to sampling (determined from the dispersion in the realizations) and the fitting-error of the observations. These values are listed in Table 6 . The observed and best-fitting model profiles at both angular resolutions are shown in Fig. 10.
A model with $\eta \simeq 0$ at all radii is a priori unlikely: previous work has found values of $0.5 \lesssim \eta \lesssim 1$ in other sources (Dolag 2006; Guidetti et al. 2008; Laing et al. 2008). The most likely explanation for the low value of $\eta$ inferred from the low-resolution image is that the electron density distribution is not well represented by a $\beta$-model (which describes a spherical and smooth distribution) at large radii. In support of this idea, Fig. 1 shows that the morphology of the X-ray emission is not spherical at large radii, but quite irregular. Croston et al. (2003) and Croston et al. (2008) pointed out that the quality of the fit of a single $\beta$-model to the X-ray surface brightness profile was poor in the outer regions, suggesting small-scale deviations in the gas distribution. The single $\beta$-model gave a better fit to the inner region of the X-ray surface brightness profile, where the polarized emission of 3C 449 can be observed at 1.25 -arcsec resolution. Our aim is to fit the $\sigma_{\mathrm{RM}}$ profiles at both resolutions with the same distribution of $n_{\mathrm{e}}(r)\left\langle B(r)^{2}\right\rangle^{1 / 2}$. In the rest of this subsection we assume that the density profile $n_{\mathrm{e}}(r)$ is still represented by the single $\beta$-model, even though we have argued that it might not be appropriate in the outer regions of the hot gas distribution. Although the resulting estimates of field strength at large radii may be unreliable, the fit is still necessary for the calculation of the outer scale described in Sect. 7.3, which depends only on the combined spatial variation of density and field strength.

The best-fitting model at 1.25 -arcsec resolution, which is characterized by a more physically reliable $\eta$, gives a very bad fit to the low resolution profile at almost all distances from the core (Fig. 10a). Conversely, the model determined at 5.5-arcsec resolution gives a very poor fit to the sharp peak in $\sigma_{\mathrm{RM}}$ observed within $20 \mathrm{kpc}$ of the nucleus at 1.25 -arcsec resolution, where the radio and X-ray data give the strongest constraints (Fig. 10b). We also verified that no single intermediate value of $\eta$ gives an adequate fit to the $\sigma_{\mathrm{RM}}$ profiles at all distances from the nucleus.

A better description of the observed $\sigma_{\mathrm{RM}}$ profile is provided by the empirical function

$$
\begin{aligned}
\left\langle B^{2}(r)\right\rangle^{1 / 2} & =B_{0}\left[\frac{n_{\mathrm{e}}(r)}{n_{0}}\right]^{\eta_{\text {int }}} & & r \leq r_{\mathrm{m}} \\
& =B_{0}\left[\frac{n_{\mathrm{e}}(r)}{n_{0}}\right]^{\eta_{\text {out }}} & & r>r_{\mathrm{m}},
\end{aligned}
$$

where $\eta_{\text {int }}, \eta_{\text {out }}$ are the inner and outer scaling index of the magnetic field and $r_{\mathrm{m}}$ is the break radius.

We fixed $\eta_{\text {int }}=1.0$ and $\eta_{\text {out }}=0.0$, consistent with our initial results, in order to reproduce both the inner sharp peak and the outer flat decline of the $\sigma_{\mathrm{RM}}$ observed at the two resolutions, keeping $r_{\mathrm{m}}$ as a free parameter. We made three sets of three-dimensional simulations for values of the outer scale $\Lambda_{\max }=205,65$ and $20 \mathrm{kpc}$. Anticipating the result of Sect. 7.3, we plot the results only for $\Lambda_{\max }=65 \mathrm{kpc}$, but the derived $\sigma_{\mathrm{RM}}$ profiles are in any case almost independent of the value 

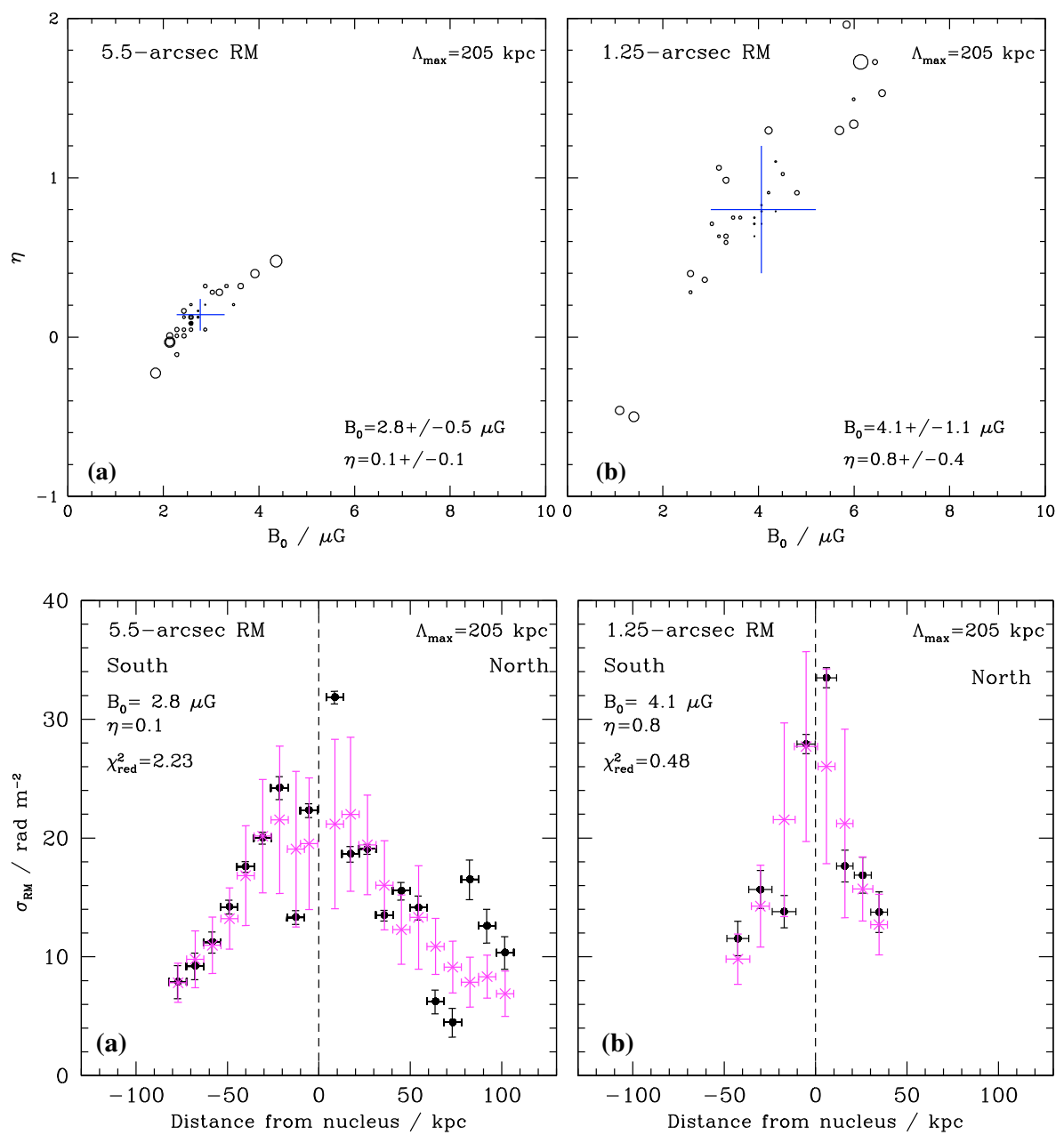

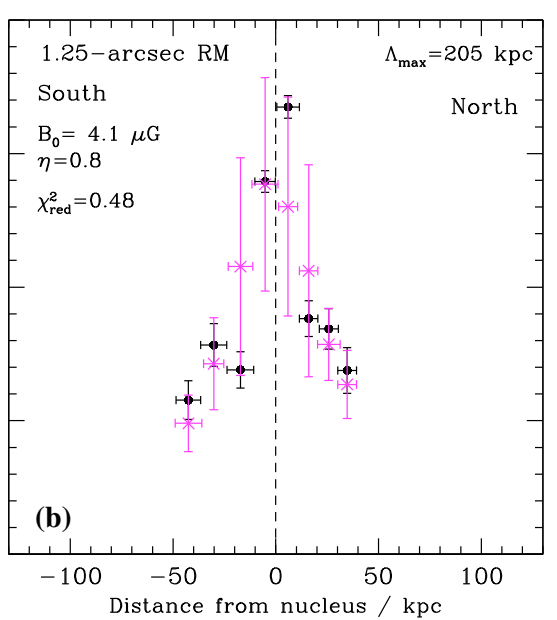

Fig. 9. a) and b) distributions of the best-fitting values of $B_{0}$ and $\eta$ from 35 sets of simulations, each covering ranges of $0.5-10 \mu \mathrm{G}$ in $B_{0}$ and $0-2$ in $\eta$, at 5.5 and 1.25 arcsec respectively. The sizes of the circles are proportional to $\chi^{2}$ for the fit and the blue crosses represent the means of the distributions weighted by $1 / \chi^{2}$. The plot shows the expected degeneracy between $B_{0}$ and $\eta$.
Fig. 10. a) Observed and synthetic radial profiles for rms Faraday $\sigma_{\mathrm{RM}}$ at $5.5 \operatorname{arcsec}$ as functions of the projected distance from the radio source centre. The outer scale is $\Lambda_{\max }=$ $205 \mathrm{kpc}$. The black points represent the data with vertical bars corresponding to the rms error of the RM fit. The magenta crosses represent the mean values from 35 simulated profiles and the vertical bars are the rms scatter in these profiles due to sampling. b) as a), but at 1.25 arcsec. of the outer scale in this range. The new simulations were made only at a resolution of 5.5 arcsec, since the larger field of view at this resolution is essential to define the change in slope of the profile.

In order to determine the best-fitting break radius, $r_{\mathrm{m}}$ in Eq. (11), we produced 35 sets of synthetic RM images for a grid of values of $B_{0}$ and $r_{\mathrm{m}}$ for each outer scale, noting the pair of values which gave the minimum unweighted $\chi^{2}$ for the $\sigma_{\text {RM }}$ profile for each set of simulations. These values are plotted in Fig. 11, which shows that there is a degeneracy between the break radius $r_{\mathrm{m}}$ and $B_{0}$. As with the similar degeneracy between $B_{0}$ and $\eta$ noted earlier, there is a clear minimum in $\chi^{2}$, and we therefore adopted the mean values of $B_{0}$ and $r_{\mathrm{m}}$ weighted by $1 / \chi^{2}$ as our best estimates of the magnetic-field parameters.

We then made 35 simulations with the best-fitting values of $B_{0}$ and $\eta$ for each outer scale and evaluated the weighted $\chi^{2}$ 's for the resulting $\sigma_{\mathrm{RM}}$ profiles. All three values of $\Lambda_{\max }$ we investigated give reasonable fits to the observed $\sigma_{\mathrm{RM}}$ profile along the whole radio source. The fit for $\Lambda_{\max }=65 \mathrm{kpc}$ is marginally better than for the other two values $\left(\chi_{\text {red }}^{2}=1.8\right)$, consistent with the results of Sect. 7.3 below. In this case the central magnetic field strength is $3.5 \pm 1.2 \mu \mathrm{G}$ and the break radius is $16 \pm 11 \mathrm{kpc}$. For the power spectrum with $\Lambda_{\max }=65 \mathrm{kpc}$ and these best-fitting parameters, we also produced three-dimensional simulations at a resolution of 1.25 arcsec. Even though the fitting procedure is based only on the low-resolution data, this model also reproduces the 1.25 -arcsec profile very well $\left(\chi_{\text {red }}^{2}=0.7\right)$. Combining the values of $\chi^{2}$ for the two resolutions, using the 1.25 -arcsec profile close to the core and the 5.5-profile at larger distances, we find $\chi_{\text {red }}^{2}=1.8$.

Figure 12 shows a comparison of the observed radial profiles for rms Faraday $\sigma_{\mathrm{RM}}$ and $\langle\mathrm{RM}\rangle$ with the synthetic ones derived for this model. The synthetic $\sigma_{\mathrm{RM}}$ profile plotted in Fig. 12 is the mean over 35 simulations and may be compared directly with the observations. In contrast, the $\langle\mathrm{RM}\rangle$ profile is derived from a single example realization. It is important to emphasize that the latter is one example of a random process, and is not expected to fit the observations; rather, we aim to compare the fluctuation amplitude as a function of position.

The values of $B_{0}$ and $\chi_{\text {red }}^{2}$ for all of the three-dimensional simulations, together with $\eta$ and $r_{\mathrm{m}}$ for the single and double power-law profiles, respectively, are summarized in Table 6.

\subsection{The outer scale of the magnetic-field fluctuations}

The theoretical RM structure function for uniform field strength, density and path length and a power spectrum with a lowfrequency cut-off should asymptotically approach a constant value $\left(2 \sigma_{\mathrm{RM}}^{2}\right.$ for a large enough averaging region) at separations $\gtrsim \Lambda_{\max }$. The observed RM structure function of the whole source is heavily modified from this theoretical one by the scaling of the electron gas density and magnetic field at large separations, which acts to suppress power on large spatial scales. In Sect. 6 we therefore limited the study of the structure function to sub-regions of 3C 449 where uniformity of field strength, density and path length (and therefore of the power-spectrum 
A\&A 514, A50 (2010)

Table 6. Results from the three-dimensional fits at both the angular resolutions of 1.25 and 5.5 arcsec.

\begin{tabular}{cccccccc}
\hline \hline $\begin{array}{c}F W H M \\
(\operatorname{arcsec})\end{array}$ & $\begin{array}{c}\Lambda_{\max } \\
(\mathrm{kpc})\end{array}$ & $B_{0}(\mu \mathrm{G})$ & $\begin{array}{c}\text { single } \eta \\
\gamma_{\text {red }}\end{array}$ & $B_{0}(\mu \mathrm{G})$ & $r_{\mathrm{m}}(\mathrm{kpc})$ & $\chi_{\text {red }}^{2}$ \\
\hline 1.25 & 205 & $4.1 \pm 1.1$ & $0.8 \pm 0.4$ & 0.48 & - & - & - \\
5.50 & 205 & $2.8 \pm 0.5$ & $0.1 \pm 0.1$ & 2.2 & $3.5 \pm 0.7$ & $17 \pm 9$ & 1.9 \\
5.50 & 65 & - & - & - & $3.5 \pm 1.2$ & $16 \pm 11$ & 1.8 \\
5.50 & 20 & - & - & - & $3.5 \pm 0.8$ & $11 \pm 8$ & 2.1 \\
\hline
\end{tabular}

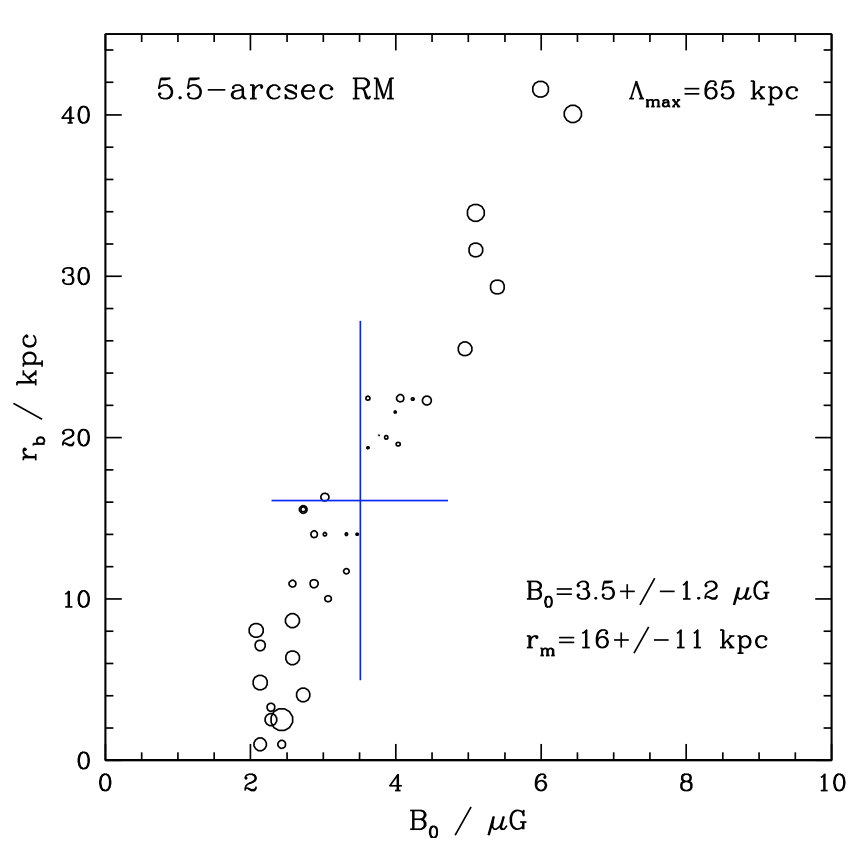

Fig. 11. Distribution of the best-fitting values of $B_{0}$ and $r_{\mathrm{m}}$ from 35 sets of simulations, each covering ranges of $0.5-10 \mu \mathrm{G}$ in $B_{0}$ and $0-50 \mathrm{kpc}$ in $r_{\mathrm{m}}$. The sizes of the circles are proportional to $\chi^{2}$ and the blue cross represents the means of the distribution weighted by $1 / \chi^{2}$. The plot shows the degeneracy between $B_{0}$ and $r_{\mathrm{m}}$ described in the text.

amplitude) is a reasonable assumption, inevitably limiting our ability to constrain the power spectrum on the largest scales.

Now that we have an adequate model for the variation of $n_{\mathrm{e}}(r)\left\langle B^{2}(r)\right\rangle^{1 / 2}$ with radius (Eq. (11)), we can correct for it to derive what we call the pseudo-structure function - that is the structure function for a power-spectrum amplitude, which is constant over the source. This can be compared directly with the structure functions derived from the Hankel transform of the power spectrum. To evaluate the pseudo-structure function, we divided the observed 5.5-arcsec RM image by the function

$$
\left[\int_{0}^{L} n_{\mathrm{e}}(r)^{2} B(r)^{2} \mathrm{~d} l\right]^{1 / 2}
$$

(Enßlin \& Vogt 2003), where the radial variations of $n_{\mathrm{e}}$ and $B$ are those of the best-fitting model (Sect. 7) and the upper integration limit $L$ has a length of 10 times the core radius. The integral was normalized to unity at the position of the radio core: this is equivalent to fixing the field and gas density at their maximum values and holding them constant everywhere in the group. The normalization of the pseudo-structure function should then be quite close to that of the two central jet regions.

The pseudo-structure function is shown in Fig. 14a together with the predictions for the BPL power spectra with $\Lambda_{\max }=$ 205, 65 and $20 \mathrm{kpc}$. As expected, the normalization of the pseudo-structure function is consistent with that of the jets (Figs. 8a and b).

The comparison between the synthetic and observed pseudostructure functions indicates firstly that they agree very well at small separations, independent of the value of $\Lambda_{\max }$. This confirms that the best BPL power spectrum found from a combined fit to all six sub-regions is a very good fit over the entire source. Secondly, despite the poor sampling on very large scales, the asymptotic values of the predicted structure functions for the three values of $\Lambda_{\max }$ are sufficiently different from each other that we can determine an approximate outer scale. The model with $\Lambda_{\max }=65 \mathrm{kpc}$ gives the best representation of the data. The fit is within the estimated errors except for a marginal discrepancy at the very largest (and therefore poorly sampled) separations. That with $\Lambda_{\max }=20 \mathrm{kpc}$ is inconsistent with the observed pseudo-structure function for any separation $\gtrsim 20$ arcsec $(\simeq 6 \mathrm{kpc})$ where the sampling is still very good, and is firmly excluded. The model with $\Lambda_{\max }=205 \mathrm{kpc}$ has slightly, but significantly too much power on large scales.

We emphasize that our estimate of the outer scale of the $\mathrm{RM}$ fluctuations is essentially independent of the functional form assumed for the variation of field strength with radius in the central region, which affects the structure function only for small separations. Our results are almost identical if we fit the fieldstrength variation with either the profile of Eq. (10) (with $\eta \approx 0$ ) or that of Eq. (11).

As for the structure functions of individual regions, the pseudo-structure function at large separations is clearly affected by poor sampling: this increases the errors, but does not produce any bias in the derived values. At large radii, however, the integral in Eq. (12) becomes small, so the noise on the RM image is amplified. This is a potential source of error, and we therefore checked our results using numerical simulations. We calculated the mean and rms structure functions for sets of realizations of RM images generated with the FARADAY code, as in Sect. 7.1, for different values of $\Lambda_{\max }$. These structure functions are plotted in Figs. $14 \mathrm{~b}$ and c.

The main difference between the model structure functions derived from simulations and the pseudo-structure functions described earlier is that the former show a steep decline in power on large scales in place of a plateau. This occurs because the smooth fall-off in density and magnetic field strength with distance from the nucleus suppresses the fluctuations in RM on large scales.

The results of the simulations confirm our analysis using the pseudo-structure function. The mean model structure function with $\Lambda_{\max }=65 \mathrm{kpc}$ again fits the data very well, except for a marginal discrepancy at the largest scales. In view of the deviations from spherical symmetry on large scales evident in the X-ray emission surrounding 3C 449 (Fig. 1), we do not regard this as a significant effect.

In order to check that the best-fitting density and field model also reproduces the data at small separations, we repeated the analysis at 1.25 -arcsec resolution. The observed pseudo-structure function is shown in Fig. 14d, together with the 

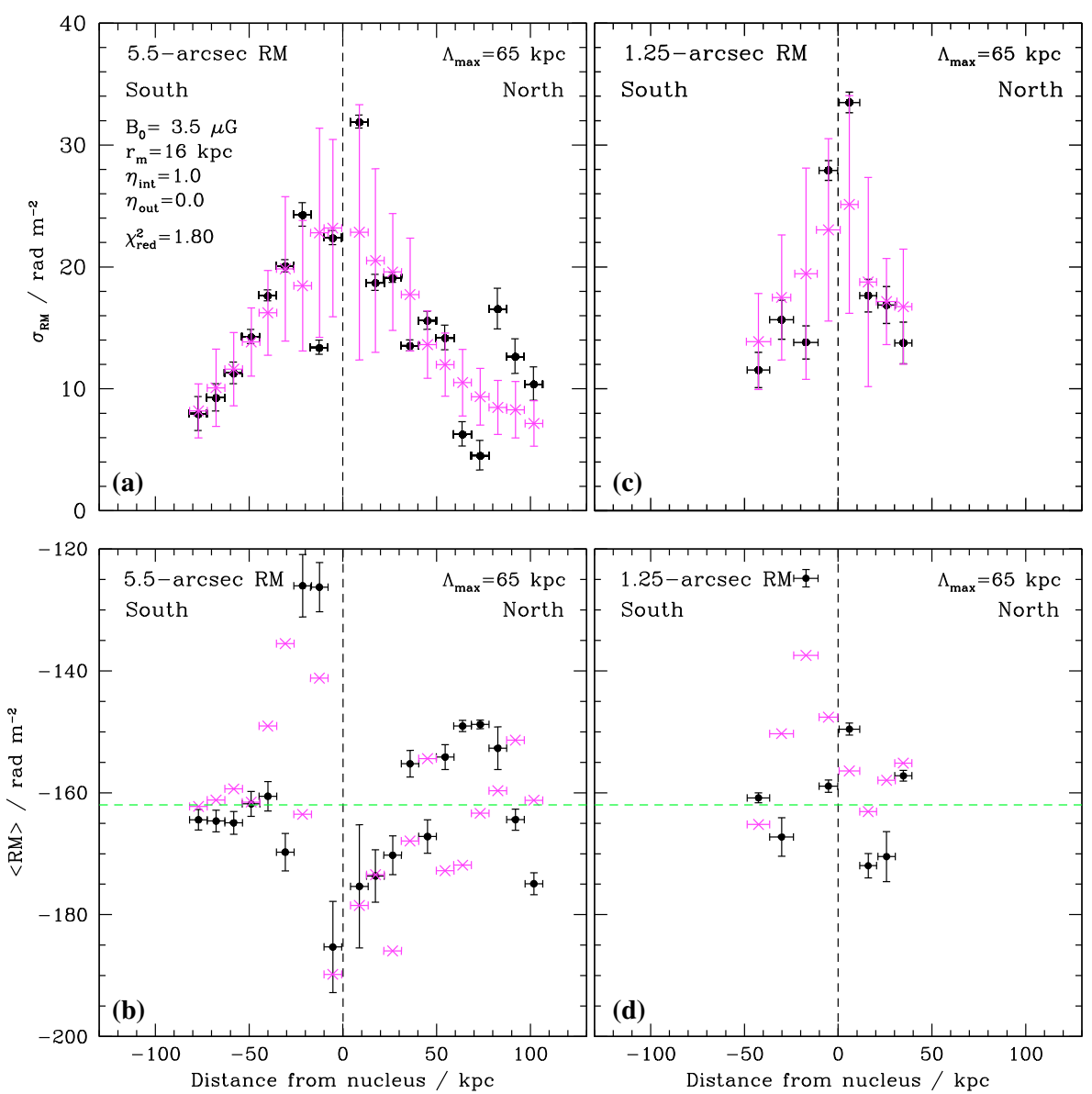

Fig. 12. Comparison between observed and synthetic profiles of rms and mean Faraday rotation at resolutions of $5.5 \operatorname{arcsec}$ (a) and b)) and $1.25 \operatorname{arcsec}(\mathbf{c})$ and $\mathbf{d})$ ). The synthetic profiles are derived from the best-fitting model with $\Lambda_{\max }=65 \mathrm{kpc}$. The black points represent the data with vertical bars corresponding to the rms fitting error. a) and c) Profiles of $\sigma_{\mathrm{RM}}$. The magenta crosses represent the mean values from 35 simulations at 5.5 -arcsec resolution, and the vertical bars are the rms scatter in these profiles due to sampling. b) and d) Profiles of $\langle\mathrm{RM}\rangle$ derived from single example realizations at 5.5 and 1.25 -arcsec resolutions. predictions for the BPL power spectrum with $\Lambda_{\max }=205,65$ and $20 \mathrm{kpc}$. The observed structure function is compared with the mean from 35 simulations with $\Lambda_{\max }=65 \mathrm{kpc}$ in Fig. 14e. In both cases, the agreement for $\Lambda_{\max }=65 \mathrm{kpc}$ is excellent.

In Fig. 13, example realizations of this model with the best-fitting field variation are shown for resolutions of 1.25 and 5.5 arcsec alongside the observed RM images.

\section{Summary and comparison with other sources}

\subsection{Summary}

In this work we have studied the structure of the magnetic field associated with the ionized medium around the radio galaxy $3 \mathrm{C} 449$. We have analysed images of linearly polarized emission with resolutions of $1.25 \operatorname{arcsec}$ and $5.5 \operatorname{arcsec} F H W M$ at seven frequencies between 1.365 and $8.385 \mathrm{GHz}$, and produced images of degree of polarization and rotation measure. The RM images at both the angular resolutions show patchy and random structures. In order to study the spatial statistics of the magnetic field, we used a structure-function analysis and performed two- and three-dimensional RM simulations. We can summarize the results as follows.

1. The absence of deviations from $\lambda^{2}$ rotation over a wide range of polarization position angle implies that a pure foreground Faraday screen with no mixing of radio-emitting and thermal electrons is a good approximation for 3C 449 (Sect. 4).

2. The dependence of the degree of polarization on wavelength is well fitted by a Burn law. This is also consistent with pure foreground rotation, with the residual depolarization observed at the higher resolution being due to unresolved
RM fluctuations across the beam (Sect. 5). There is no evidence for a detailed correlation of radio-source structure with either RM or depolarization.

3. There is no obvious anisotropy in the RM distribution, consistent with our assumption that the magnetic field is an isotropic, Gaussian random variable.

4. Our best estimate for the Galactic contribution to the RM of $3 \mathrm{C} 449$ is a constant value of $-160.7 \mathrm{rad} \mathrm{m}^{-2}$ (Sect. 4.2).

5. The RM structure functions for six different regions of the source are consistent with the hypothesis that only the amplitude of the RM power spectrum varies across the source.

6. A broken power-law spectrum of the form given in Eq. (9) with $q_{1}=2.07, q_{\mathrm{h}}=2.98, f_{\mathrm{b}}=0.031 \mathrm{arcsec}^{-1}$ and $f_{\max }=1.67 \operatorname{arcsec}^{-1}$ (corresponding to a spatial scale $\Lambda_{\text {min }}=$ $0.2 \mathrm{kpc}$ ) is consistent with the observed structure functions and depolarizations for all six regions. No single power law provides a good fit to all of the structure functions.

7. The high-frequency cut-off in the power spectrum is required to model the depolarization data.

8. The profiles of $\sigma_{\mathrm{RM}}$ strongly suggest that most of the fluctuating component of RM is associated with the intra-group gas, whose core radius is comparable with the characteristic scale of the profile (Sect. 4.1). The symmetry of the profile is consistent with the idea that the radio source axis is close to the plane of the sky.

9. We therefore simulated the RM distributions expected for an isotropic, random magnetic field in the hot plasma surrounding 3C 449, assuming the density model derived by Croston et al. (2008).

10. These three-dimensional simulations show that the dependence of magnetic field on density is best modelled by a 

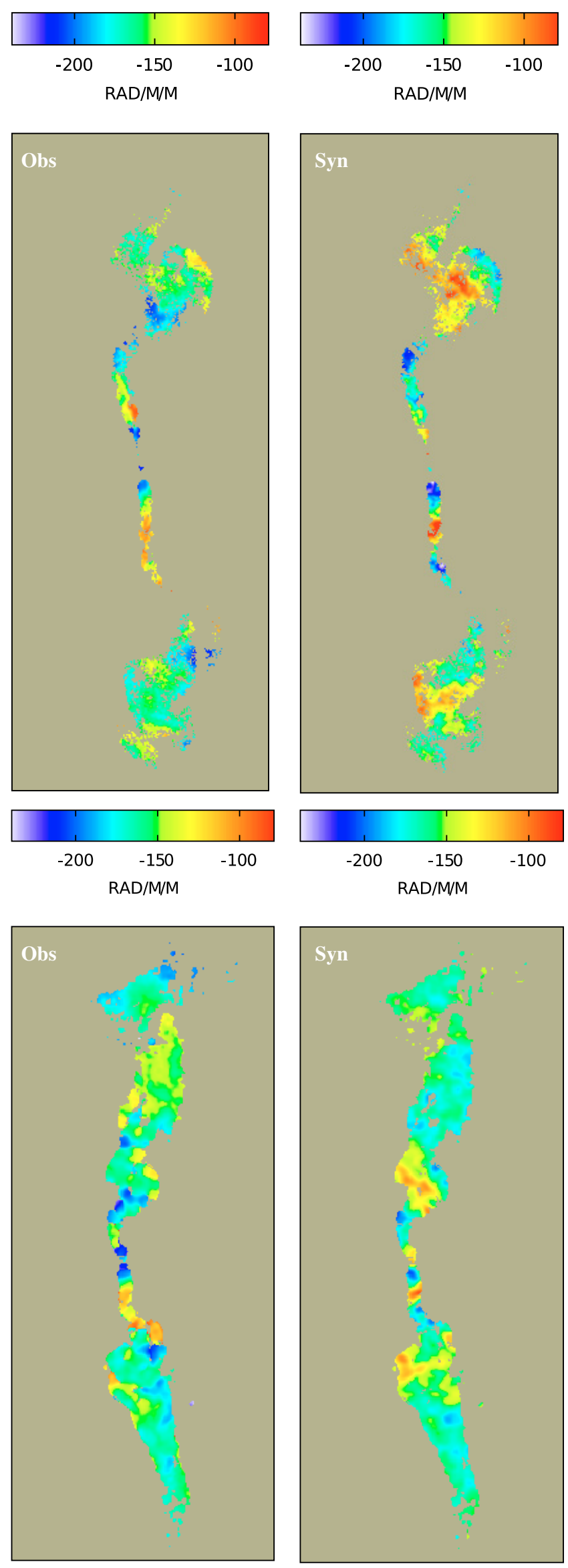

Fig. 13. Comparison of observed and representative synthetic distribution of Faraday $\mathrm{RM}$ at 1.25 arcsec and 5.5 arcsec. The synthetic images have been produced for the best-fitting model with $\Lambda_{\max }=65 \mathrm{kpc}$. The colour scale is the same for all displays. 
D. Guidetti et al.: Structure of the magnetoionic medium around the Fanaroff-Riley Class I radio galaxy 3C 449
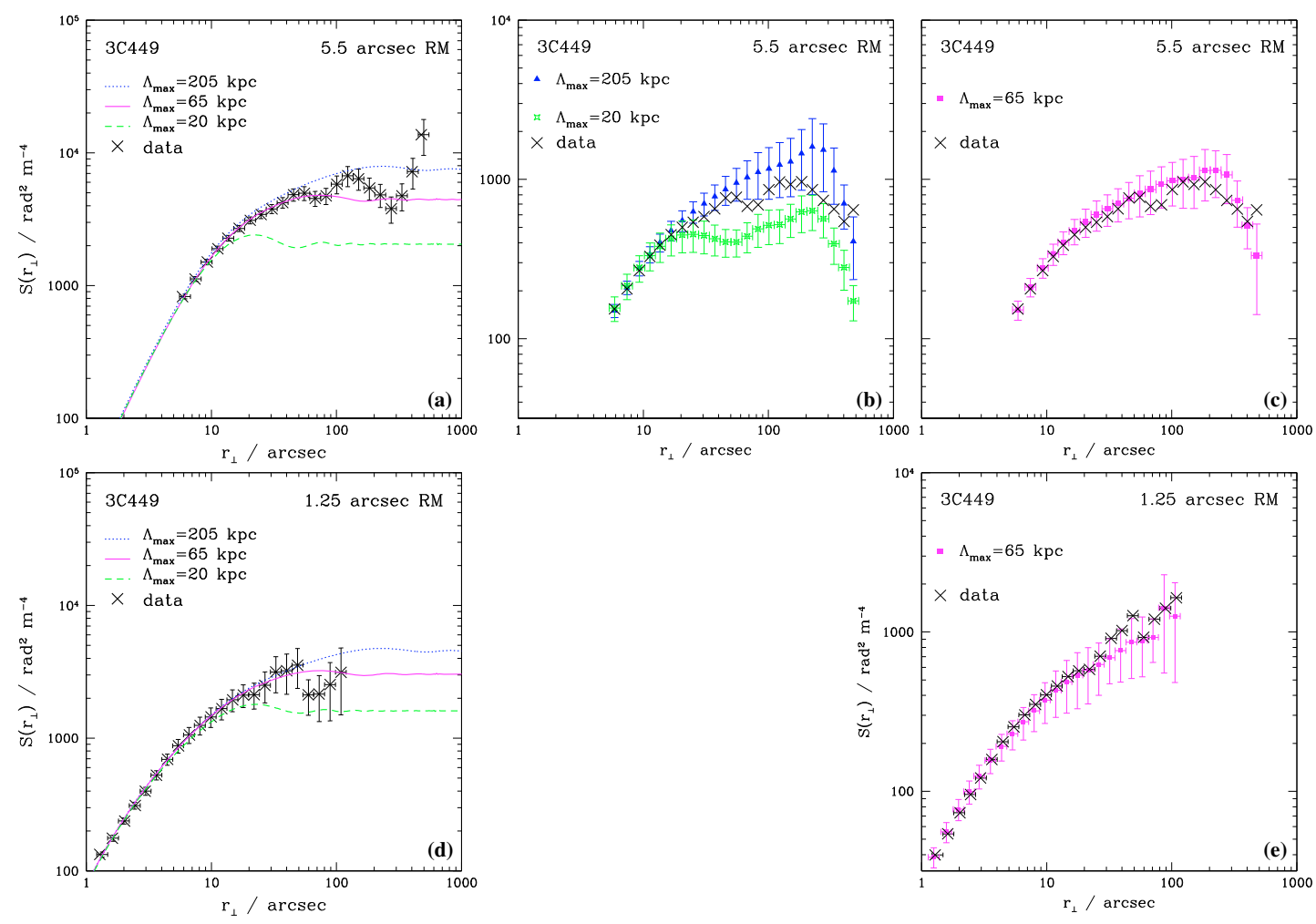

Fig. 14. a) Comparison of the observed pseudo-structure function of the 5.5 -arcsec RM image as described in the text (points) with the predictions of the BPL model (curves, derived using a Hankel transform). The predicted curves are for $\Lambda_{\max }=205$ (blue dotted), 65 (continuous magenta) and $20 \mathrm{kpc}$ (green dashed). b) and c) structure functions of the observed (points) and synthetic 5.5-arcsec RM images produced with the $\Lambda_{\max }=20520 \mathrm{kpc}$ and $\Lambda_{\max }=65 \mathrm{kpc}$, respectively. d) and e) as a) and c) respectively, but at 1.25 -arcsec resolution. The error bars are the rms from 35 structure functions at a given $\Lambda_{\max }$, in a) and d) are representative error bars from the model with $\Lambda_{\max }=65 \mathrm{kpc}$.

broken power-law function with $B(r) \propto n_{\mathrm{e}}(r)$ close to the nucleus and $B(r) \approx$ constant at larger distances.

11. With this density model, our best estimate of the central magnetic field strength is $B_{0}=3.5 \pm 1.2 \mu \mathrm{G}$.

12. Assuming these variations of density and field strength with radius, a structure-function analysis can be used to estimate the outer scale $\Lambda_{\max }$ of the magnetic-field fluctuations. We find excellent agreement for $\Lambda_{\max } \approx 65 \mathrm{kpc}\left(f_{\min }=\right.$ $\left.0.0053 \operatorname{arcsec}^{-1}\right)$.

\subsection{Comparison with other sources}

Our results are qualitatively similar to those of Laing et al. (2008) on 3C 31. The maximum RM fluctuation amplitudes are similar in the two sources, as are their environments. For spherically-symmetric gas density models, the central magnetic fields are almost the same: $B_{0} \approx 2.8 \mu \mathrm{G}$ for $3 \mathrm{C} 31$ and $3.5 \mu \mathrm{G}$ for $3 \mathrm{C} 449$. Both results are consistent with the idea that the RM fluctuation amplitude in galaxy groups and clusters scales roughly linearly with density, ranging from a few $\mathrm{rad} \mathrm{m}^{-2}$ in the sparsest environments (e.g. NGC 315; Laing et al. 2006), through intermediate values $\approx 30-100 \mathrm{rad} \mathrm{m}^{-2}$ in rich groups such as 3C 31 and 3C 449 to $\sim 10^{4} \mathrm{rad} \mathrm{m}^{-2}$ in the centres of clusters with cool cores.

The RM distribution of 3C 31 is asymmetrical, the northern (approaching) side of the source showing a much lower fluctuation amplitude, consistent with the inclination of $\approx 50^{\circ}$ estimated by Laing \& Bridle (2002). Detailed modelling of the RM profile led Laing et al. (2008) to suggest that there is a cavity in the X-ray gas, but this would have to be significantly larger than the observed extent of the radio lobes and is, as yet, undetected in X-ray observations. A broken power-law scaling of magnetic field with density, similar to that found for 3C 449 in the present paper, would also improve the fit to the $\sigma_{\mathrm{RM}}$ profile for 3C 31; alternatively, the effects of cavities around the inner lobes and spurs of 3C 449 might be significant. Deeper X-ray observations of both sources are needed to resolve this issue. In neither case is the magnetic field dynamically important: for $3 \mathrm{C} 449$ we find that the ratio of the thermal and magnetic-field pressures is $\approx 30$ at the nucleus and $\approx 400$ at the core radius of the group gas, $r_{\mathrm{c}}=19 \mathrm{kpc}$.

The magnetic-field power spectrum in both sources can be fit by a broken power-law form. The low-frequency slopes are 2.1 and 2.3 for 3C 449 and 3C 31 respectively. In both cases, the power spectrum steepens at higher spatial frequencies, but for 3C 31 a Kolmogorov index (11/3) provides a good fit, whereas we find that the depolarization data for 3C 449 require a cutoff below a scale of $0.2 \mathrm{kpc}$ and a high-frequency slope of 3.0. The break scales are $\approx 5 \mathrm{kpc}$ for $3 \mathrm{C} 31$ and $\approx 11 \mathrm{kpc}$ for $3 \mathrm{C} 449$. It is important to note that the simple parametrized form of the power spectrum is not unique, and that a smoothly curved function would fit the data equally well.

The gas-density structure on large scales in the $3 \mathrm{C} 31$ group is uncertain, so Laing et al. (2008) could only give a rough lower limit to the outer scale of magnetic-field fluctuations, $\Lambda_{\max } \gtrsim$ $70 \mathrm{kpc}$. For $3 \mathrm{C} 449$, we find $\Lambda_{\max } \approx 65 \mathrm{kpc}$. The projected distance between 3C 449 and its nearest neighbour is $\approx 33 \mathrm{kpc}$ (Birkinshaw et al. 1981), similar to the scale on which the jets first bend through large angles (Fig. 1). As in 3C 31, it is plausible that the outer scale of magnetic-field fluctuations is set by interactions with companion galaxies in the group. 
Acknowledgements. This work is part of the "Cybersar" Project, which is managed by the COSMOLAB Regional Consortium with the financial support of the Italian Ministry of University and Research (MUR), in the context of the "Piano Operativo Nazionale Ricerca Scientifica, Sviluppo Tecnologico, Alta Formazione (PON 2000-2006)". We thank Luigina Feretti for providing the VLA data for 3C 449, Greg Taylor for the use of his rotation measure code and Marco Bondi for many helpful comments. We also acknowledge the use of HEALPIX package (http://healpix.jpl.nasa.gov) and the provision of the models of Dineen \& Coles (2005) in HEALPIX format.

\section{References}

Andernach, H., Feretti, L., Giovannini, G., et al. 1992, A\&A, 93, 331 Balbus, S. A. 2000, ApJ, 534, 420

Birkinshaw, M., Laing, R. A., \& Peacock, J. A. 1981, MNRAS, 197, 253

Bogdanović, T., Reynolds, C. S., Balbus, S. A., \& Parrish, I. J. 2009, ApJ, 704, 211

Brunetti, G., Setti, G., Feretti, \& Giovannini, L. G. 2001, MNRAS, 320, 365

Burn, B. J. 1966, MNRAS, 133, 67

Carilli, C. L., \& Taylor, G. B. 2002, ARA\&A, 40, 319

Cavaliere, A., \& Fusco-Femiano, R. 1976, A\&A, 49, 137

Clarke, T. E., Kronberg, P. P., \& Böhringer, H. 2001, ApJ, 547, L111

de Vaucouleurs, G., de Vaucouleurs, A., Corwin, Jr., et al. 1991, Third Reference Catalogue of Bright Galaxies (New York: Springer)

Croston, J. H., Hardcastle, M. J., Birkinshaw, M., \& Worrall, D. M. 2003, MNRAS, 346, 1041

Croston, J. H., Hardcastle, M. J., Birkinshaw, M., \& Laing, R. A. 2008, MNRAS, 386, 1709

Dineen, P., \& Coles, P. 2005, MNRAS, 362,403

Dolag, K. 2006, Astron. Nachr., 327, 575

Dolag, K., Schindler, S., Govoni, F., \& Feretti, L. 2001, A\&A, 378, 777

Dursi, L. J., \& Pfrommer, C. 2008, ApJ, 677, 993

Eilek, J. A., \& Owen, F. N. 2002, ApJ, 567, 202

Enßlin, T. A., \& Vogt, C. 2003, A\&A, 401, 835

Fanaroff, B. L., \& Riley, J. M. 1974, MNRAS, 167, 31

Feretti, L., Dallacasa, D., Giovannini, G., \& Tagliani, A. 1995, A\&A, 302, 680
Feretti, L., Perley, R., Giovannini, G., \& Andernach, H. 1999a, A\&A, 341, 29 Feretti, L., Dallacasa, D., Govoni, F., et al. 1999b, A\&A, 344, 472 Govoni, F., \& Feretti, L. 2004, Inter. J. Mod. Phys. D 13, 1549

Govoni, F., Taylor, G. B., Dallacasa, D., Feretti, L., \& Giovannini, G. 2001, A\&A, 379, 807

Govoni, F., Murgia, M., Feretti, L., et al. 2006, A\&A, 460, 425

Guidetti, D., Murgia, M., Govoni, F., et al. 2008, A\&A, 483, 699

Hardcastle, M. J., Worrall, D. M., \& Birkinshaw, M. 1998, MNRAS, 296, 1098

Kim, K. T., Kronberg, P. P., Dewdney, P. E., \& Landecker, T. L. 1990, ApJ, 355, 29

Kim, K. T., Kronberg, P. P., \& Tribble, C. 1991, ApJ, 379, 80

Laing, R. A. 1984, in Physics of Energy Transport in Extragalactic Radio Sources, ed. A. H. Bridle, \& J. A. Eilek, NRAO Workshop, 9, 90

Laing, R. A., \& Bridle, A. H. 2002, MNRAS, 336, 328

Laing, R. A., Canvin, J. R., Cotton, W. D., \& Bridle, A. H. 2006, MNRAS, 368, 48

Laing, R. A., Bridle, A. H., Parma, P., \& Murgia, M. 2008, MNRAS, 391, 521

Lawler, J. M., \& Dennison, B. 1982, ApJ, 252, 81

Minter, A. H., \& Spangler, S. R. 1996, ApJ, 467, 37

Murgia, M., Govoni, F., Feretti, L., et al. 2004, A\&A, 424, 429

O’Neill, S. M., De Young, D. S., \& Jones, T. W. 2009, ApJ, 694, 1317

Perley, R. A., Willis, A. G., \& Scott, J. S. 1979, Nature, 281, 437

Perley, R. A., Bridle, A. H., \& Willis, A. G. 1984, ApJS, 54, 291

Pollack, L. K., Taylor, G. B., \& Allen, S. W. 2005, MNRAS, 359, 1229

Simard-Normandin, M., Kronberg, P. P., \& Button, S. 1981, ApJS, 45, 97

Simonetti, J. H., Cordes, J. M., \& Spangler, S. R. 1984, ApJ, 284, 126

Taylor, G. B., \& Perley, R. A. 1993, ApJ, 416, 554

Taylor, G. B., Fabian, A. C., \& Allen, S. W. 2002, MNRAS, 334, 769

Ulvestad, J. S., Perley, R. A., \& Chandler, C. J. 2009, The Very Large Array observational status summary, http://www.vla.nrao.edu/astro/guides/ vlas/current/vlas.html

Vallée, J. P., MacLeod, M. J., \& Broten, N. W. 1986, A\&A, 156, 386

Vogt, C., \& Enßlin, T. A. 2003, A\&A, 412, 373

Vogt, C., \& Enßlin, T. A. 2005, A\&A, 434, 67

Wardle, J. F. C., \& Kronberg, P. P. 1974, ApJ, 194, 249

Zwicky, F., \& Kowal, C. T. 1968, Catalogue of Galaxies and of Clusters of Galaxies, California Institute of Technology, Pasadena, 6 